\title{
LISTS OF SPECIES
}

\section{Checklist of polychaete species from Paraná State (Southern Brazil)}

\author{
Paulo Cunha Lana ${ }^{1}$ \\ Cinthya S. G. Santos ${ }^{1}$ \\ André R. S. Garraffoni \\ Verônica M. Oliveira ${ }^{1}$ \\ Vasily Radashevsky ${ }^{3}$ \\ ${ }^{1}$ Centro de Estudos do Mar, Universidade Federal \\ do Paraná, Caixa Postal 50002, CEP 83255-000, \\ Pontal do Sul, Pontal do Paraná, PR, Brazil. E- \\ mail: lana@ufpr.br
}

${ }^{2}$ Universidade Estadual de Campinas, Instituto de Biologia, Departamento de Zoologia, Caixa Postal 6109, CEP 13083-970, Campinas, SP, Brazil.

${ }^{3}$ Institute of Marine Biology, Vladivostok, Russia.

\begin{abstract}
A checklist of polychaete species recorded from Paraná State (Southern Brazil, 25 ${ }^{\circ} 10^{\prime}-25^{\circ} 58^{\prime} \mathrm{S} /$ $47^{\circ} 59^{\prime}-48^{\circ} 35^{\prime} \mathrm{W}$ ), including estuarine areas from Paranaguá and Guaratuba Bays and shallow continental shelf bottoms, is reported. Altogether 44 families, 164 genera, and 259 valid species are currently known from the area. The list, which provides a synthesis of the regional taxonomical work carried out until now, can serve as a baseline survey for future studies.
\end{abstract}

\section{Introduction}

This checklist provides an updated overview of the polychaete species recorded from Paranaguá Bay and adjacent areas (S Brazil), based on literature records, including unpublished theses or technical reports.

Families, genera and species names are arranged in alphabetical order, to make easier the search for a given taxon. Original descriptions plus all the local taxonomical references are listed for all species. Ecological literature which mentions polychaete species is listed in the section "additional references".

No specimens were re-examined, but we are aware that a number of the reported records need to be confirmed, since misidentifications may have happened in the original descriptions. Whenever we have good evidence that species names in published or unpublished records are wrong or inadequate, we point out the need for revisionary work.

Species names reported upon in this checklist are a result of taxonomical and ecological surveys carried out in Paraná State, at the southern Brazilian coast, since 1981. Sampling areas (Figure 1) included the estuarine environments of Paranaguá $\left(25^{\circ} 30^{\prime} \mathrm{S}-48^{\circ} 20^{\prime} \mathrm{W}\right)$ and Guaratuba bays $\left(25^{\circ} 52^{\prime} \mathrm{S}-48^{\circ} 34^{\prime} \mathrm{W}\right)$ and the outer, inner and midcontinental shelf off Paraná $\left(25^{\circ} 10^{\prime}-\right.$ $25^{\circ} 58^{\prime} \mathrm{S} / 47^{\circ} 59^{\prime}-48^{\circ} 35^{\prime} \mathrm{W}$ ).

Most of the reported material is currently deposited at the Centro de Estudos do Mar, Universidade Federal do Paraná (MCEM - BPO), in Pontal do Sul, Paraná (Curator: Paulo da Cunha Lana: lana@ufpr.br) and Museu de Zoologia from Universidade de São Paulo (MZUSP). Whenever available, deposit numbers are provided for each species. This collection will be transferred to the Museu de Zoologia from Universidade de São Paulo (MZUSP) by mid-2007. Some specimens were also deposited in National Museum of Natural History, Smithsonian Institution, Washington, D.C., U.S.A (USNM), Senckenberg Museum, Frankfurt am Main, Germany (SMF), Institute of Marine Biology, Vladivostok, Russia (IMBV) and Zoologische Sammlung der Universität Rostock, Germany (ZSRO). 


\section{LISTS OF SPECIES}

\section{Systematic checklist}

\section{Acoetidae Kinberg, 1858}

Eupanthalis kinbergi McIntosh, 1879

Eupanthalis kinbergi McIntosh, 1879: 313.

Eupanthalis kinbergi — Lana, 1987: 1061, table 1.

Panthalis oerstedi Kinberg, 1855

Panthalis oerstedi Kinberg, 1855: 387.

Panthalis oerstedi — Lana, 1984: 26; 1987: 1061, table 1.

\section{Ampharetidae Malmgren, 1867}

Isolda pulchella Fritz Müller, 1858

Isolda pulchella Fritz Müller, 1858: 218, figs. 26-27.

Isolda pulchella - Nonato, 1981: 202, fig. 220; Blankensteyn and Moura, 2002: 718, table 2; Amaral and Nallin 2005: 48, table 2.

Deposit number - MZUSP 316.

Melinna cristata (Sars, 1851)

Sabellides cristata Sars, 1851: 205-206.

Melinna cristata - Attolini, 2002: 23, figs. 8, 9, 11, 12, tables 4, 8.

Obs: This is probably a misidentification and this material needs to be revised.

\section{Amphinomidae Savigny in Lamarck, 1818}

Amphinome rostrata (Pallas, 1766)

Aphrodita rostrata Pallas, 1766: 106.

Amphinome rostrata - Lana, 1987: 1061, table 1.

Hipponoa gaudichaudi agulhana Day, 1967

Hiponoa gaudichaudi agulhana Day, 1967: 122, fig. 31 a-e.

Hiponoa gaudichaudi agulhana - Lana, 1984: 48; 1987: 1061, table 1.

Deposit number - MCEM - BPO 1480

Linopherus ambigua (Monro, 1933)

Eurythoe ambigua Monro, 1933: 6, fig. 2.

Linopherus ambigua - Lana, 1984: 50; 1987: 1061, table 1.

Deposit number - MCEM - BPO 1315, 1481, 1482.

Paramphinome besnardii Temperini, 1981

Paramphinome besnardii Temperini, 1981: 14, figs. 31-35.

Paramphinome besnardii — Lana, 1984: 50; 1987: 1061, table 1.

\section{Aphroditidae Malmgren, 1867}

Aphrodita aculeata Linnaeus, 1761

Aphrodita aculeata Linnaeus, 1761: 509.

Aphrodita aculeata - Rullier and Amoureux, 1979: 146.

Aphrodita longicornis Kinberg, 1855

Aphrodita longicornis Kinberg, 1855: 382.

Aphrodita longicornis - Nonato, 1981: 26; Lana, 1984: 17; 1987: 1061, table 1.

Aphroditella alta (Kinberg, 1855)

Aphrodita alta Kinberg, 1855: 381.

Aphrodita alta - Rullier and Amoureux, 1979: 146.

Aphroditella alta — Lana, 1987: 1061, table 1. 


\section{LISTS OF SPECIES}

Laetmonice parva Amaral and Nonato, 1982

Laetmonice parva Amaral and Nonato, 1982: 15, figs. 16-23.

Laetmonice parva - Lana, 1987: 1061, table 1.

\section{Capitellidae Grube, 1862}

Capitella capitata (Fabricius, 1780)

Lumbricus capitatus Fabricius, 1780: 279.

Capitella capitata - Bolívar, 1990: 59, figs. 43a-d; Blankensteyn and Moura, 2002: 718, table 2;

Amaral and Nallin, 2005: 48, table 2.

Deposit number - MCEM - BPO 542-544.

Obs: This is probably a misidentification and this material needs to be revised.

Heteromastus similis Southern, 1921

Heteromastus similis Southern, 1921: 640.

Heteromastus similis — Bolívar, 1990: 60, figs. 45a-c; Blankensteyn and Moura, 2002: 718, table 2;

Amaral and Nallin, 2005: 48, table 2.

Deposit number - MCEM - BPO 546; 547.

Mediomastus californiensis Hartman, 1947

Mediomastus californiensis Hartman, 1947: 264, p1. 26, figs. 64-65.

Mediomastus californiensis - Bolívar, 1990: 60, fig. 44a-b.

Deposit number - MCEM - BPO 545.

Obs: This is probably a misidentification and this material needs to be revised.

Notomastus hemipodus Hartman, 1947

Notomastus hemipodus Hartman, 1947: 424, pl. 48, figs. 1-5.

Notomastus hemipodus — Bolívar, 1990: 63, fig. 47a-d.

Deposit number - MCEM - BPO 582-584.

Notomastus latericeus Sars, 1851

Notomastus latericeus Sars, 1851: 199.

Notomastus latericeus - Amaral et al., 2004: 200, appendix 3.

Notomastus lobatus Hartman, 1947

Notomastus lobatus Hartman, 1947: 415, Pl. 51, figs. 1-5.

Notomastus lobatus - Bolívar, 1990: 61, fig. 46a-d.

Deposit number - MCEM - BPO 549-552; 555-580.

\section{Cirratulidae Ryckholdt, 1851}

Caulleriella alata (Southern, 1914)

Chaetozone alata Southern, 1914: 112, pl. 12, fig. 27 a-d.

Caulleriella alata - Bolívar, 1990: 55, fig. 41a-c.

Deposit number - MCEM - BPO 539.

Dodecaceria Oersted, 1843

Dodecaceria $\mathrm{sp}$.

Deposit number - MZUSP 100.

Tharyx dorsobranchialis (Kirkegaard, 1959)

Cirratulus dorsobranchialis Kirkegaard, 1959: 34, figs. 2-3.

Tharyx dorsobranchialis - Bolívar, 1990: 51, fig. 37a-e.

Deposit number - MCEM - BPO 524-528.

\section{Chaetopteridae Malmgren, 1867}

Chaetopterus Cuvier, 1827

Chaetopterus sp. 


\section{LISTS OF SPECIES}

Chaetopterus variopedatus - Camargo, 1955: 61, figs. 1, 2; Nonato, 1981: 173. Not Renier, 1804.

Obs - This is most probably a misidentification. The original specimens could not be retrieved or localized.

Mesochaetopterus xerecus Petersen and Fanta, 1969

Mesochaetopterus xerecus Petersen and Fanta, 1969: 120, figs. 1-7.

Spiochaetopterus nonatoi Bhaud and Petti, 2001

Spiochaetopterus nonatoi Bhaud and Petti, 2001: 226, figs. 2, 3, 4 a-e.

Deposit number - MZUSP 267.

\section{Cossuridae Day, 1963}

Cossura Webster and Benedict, 1887

Cossura sp.

Deposit number - MCEM - BPO 1476.

\section{Dorvilleidae Chamberlin, 1919}

Pettiboneia sanmatiensis Orensanz, 1973

Pettiboneia sanmatiensis Orensanz, 1973: 338, figs. 1-8, pl. 5.

Pettiboneia sanmatiensis - Lana, 1987: 1061, table 1.

Protodorvillea kefersteini (McIntosh, 1869)

Dorvillea kefersteini McIntosh, 1869: 417.

Protodorvillea keferstein - Lana, 1987: 1061, table 1.

Schistomeringos rudolphi (delle Chiaje, 1828)

Nereis rudolphi delle Chiaje, 1828: 176.

Schistomeringos rudolphi - Jumars, 1974: 103, fig. 1; Lana, 1984: 195, fig. 213; 1987: 1061, table 1.

\section{Eunicidae Berthold, 1827}

Eunice cariboea Grube, 1856

Eunice cariboea Grube, $1856: 57$.

Eunice cariboea — Lana, 1984: 180, figs. 195-196; 1987: 1061, table 1.

Deposit number - MZUSP 101.

Eunice rubra Grube, 1856

Eunice rubra Grube, 1856: 59.

Eunice rubra - Lana, 1984: 183, fig. 198; 1987: 1061, table 1.

Deposit number - MCEM - BPO 1444.

Eunice unifrons (Verrill, 1900)

Leodice unifrons Verrill, 1900: 644.

Eunice cf. unifrons - Attolini, 2002: 24, figs. 8, 9, 11, 12, tables 4, 8.

Eunice vittata (delle Chiaje, 1829)

Nereis vittata delle Chiaje, 1829: 195.

Eunice vittata - Lana, 1984: 181, fig. 197; 1987: 1061, table 1.

Marphysa kinbergi McIntosh, 1910

Marphysa kinbergi McIntosh, 1910: 451, fig. 9.

Marphysa kinbergi — Lana, 1984: 185, figs. 199-200; 1987: 1061, table 1.

Marphysa sanguinea (Montagu, 1815)

Nereis sanguinea Montagu, 1815: 20.

Marphysa sanguinea — Lana, 1984: 187, fig. 201; 1987: 1061, table 1.

Nematonereis schmardae McIntosh, 1885

Nematonereis schmardae McIntosh, 1885: 262.

Nematonereis schmardae — Lana, 1987: 1061, table 1 


\section{LISTS OF SPECIES}

Deposit number - MCEM - BPO 1459; 1464.

Eulepethidae Chamberlin, 1919

Grubeulepis bracteata Nonato, 1981 nomen nudum Grubeulepis bracteata Nonato, 1981: 70, figs. 76-84 (Original name in an upublished thesis).

Grubeulepis mexicana (Berkeley and Berkeley, 1969)

Eulepethus mexicanus Berkeley and Berkeley, 1939: 328, figs 4-7.

Deposit number - MCEM - BPO 1470.

\section{Flabelligeridae Saint-Joseph, 1894}

Piromis roberti (Hartman, 1951)

Semiodera roberti Hartman, 1951: 99, pl. 26, figs. 1-4.

Piromis roberti - Bolívar, 1990: 91, pl. 26, fig. 70a-d.

Deposit number - MCEM - BPO 716-717.

Obs: This is probably a misidentification and this material needs to be revised.

\section{Glyceridae Grube, 1850}

Glycera americana Leidy, 1855

Glycera americana Leidy, 1855: 147, figs. 49-50.

Glycera americana - Lana 1984: 92, figs. 84-85; 1987: 1061, table 1.

Deposit number - MCEM - BPO 1234-1240.

Glycera oxycephala Ehlers, 1887

Glycera oxycephala Ehlers, 1887: 121, figs 7-11.

Glycera cf. oxycephala - Lana, 1984: 94, figs. 86-87.

Hemipodia californiensis (Hartman, 1938)

Hemipodus californiensis Hartman, 1938: 93, figs. 2-7.

Hemipodus olivieri Orensanz and Gianuca, 1974: 11, figs. 5, 8, 9.

Hemipodus olivieri - Lana, 1984: 91, figs. 82-83; Blankensteyn and Moura, 2002: 718, table 2;

Böggemann, 2002: 82, figs. 133-135; Amaral and Nallin, 2005: 48, table 2.

Hemipodia californiensis - Böggemann 2002: 82, figs. 133-135.

Deposit number - MCEM - BPO 1241-1244.

Hemipodia pustatula (Friedrich, 1956)

Hemipodus pustatula Friedrich, 1956; 61.

Hemipodia pustatula - Böggemann, 2002: 83, figs. 136-138

Hemipodia simplex (Grube, 1857)

Glycera simplex Grube, 1857: 177.

Hemipodus rotundus Nonato, 1981: 104, figs. 125-128; — Lana, 1987: 1061, table 1.

Hemipodia simplex — Böggemann, 2002: 80, figs. 127-129.

\section{Goniadidae Kinberg, 1865}

Bathyglycinde profunda (Hartman and Fauchald, 1971)

Glycinde profunda Hartman and Fauchald, 1971: 74-76, pl. 4, figs c-e.

Bathyglycinde profunda - Amaral et al., 2004: 202, appendix 3; Rizzo and Amaral, 2004b: p. 938, figs. 1-21, table 1; Böggemann 2005: 93, figs. 49-50.

Goniada brunnea Treadwell, 1906

Goniada brunnea Treadwell, 1906: 1174, figs. 67-70.

Goniada brunnea — Lana, 1984: 102, figs. 97-99; 1987: 1061, table 1.

Deposit number - MCEM - BPO 1249; 1250; 1252. 


\section{LISTS OF SPECIES}

Goniada crudelis (Kinberg, 1865)

Lacharis crudelis Kinberg, 1865: 247

Goniada crudelis - Böggemann, 2005: 98, figs. 53-54.

Goniada emerita Audouin and Milne-Edwards, 1833

Goniada emerita Audouin and Milne-Edwards, 1833: 258.

Goniada emerita - Lana, 1984: 96, figs. 88-90; 1987: 1061, table 1.

Deposit number - MCEM - BPO 1255; 1556; 1571-1575.

Goniada echinulata Grube, 1870

Goniada echinulata Grube, 1870: 67.

Goniada littorea Hartman 1950: 23, figs. 1-10.

Goniada littorea - Lana, 1984: 98, figs. 91-93; Blankensteyn and Moura, 2002: 718, table 2; Amaral et al., 2004: 202, appendix 3; Böggemann, 2005: 69, figs. 35-36.

Deposit number - MCEM - BPO 1246; 1247; 1253; 1254.

Goniada maculata Oersted, 1843

Goniada maculata Oersted, 1843: 33.

Goniada maculata — Lana, 1984: 100, figs. 94-96; 1987: 1061, table 1; Blankensteyn and Moura, 2002: 718 , table 2.

Deposit number - MCEM - BPO 1557; 1561; 1567; 1568; 1578; 1581.

Identification not confirmed by Böggemann (2005).

Goniada virgini Kinberg, 1865

Goniada virgini Kinberg, 1865: 247

Goniada virgini - Böggemann, 2005: 93, figs. 49-50.

Goniadella revizee Rizzo and Amaral, 2004a

Goniadella revizee Rizzo and Amaral, 2004: 52, Figs. 4-6, table 2;

Goniadella revizee - Amaral et al., 2004: 202, appendix 3.

Goniadides carolinae Day, 1973

Goniadides carolinae Day, 1973: 48, fig. 7a-h.

Goniadides carolinae - Lana, 1984: 103, fig. 100; 1987: 1061, table 1; Amaral and Nallin, 2005: 48, table 2; Böggemann, 2005: 183, figs. 106-107.

Deposit number - MCEM - BPO 1573; 1577.

Glycinde multidens Müller, 1858

Glycinde multidens Müller, 1858: 214, est. 6, figs. 4-6.

Glycinde multidens - Nonato, 1981: 106, figs. 134-139; Lana, 1984: 104, figs. 101-104; 1987: 1061, table 1; Blankensteyn and Moura, 2002: 718, table 2; Amaral and Nallin, 2005: 48, table 2; Böggemann, 2005: 216, figs. 128-129.

Deposit number - MCEM - BPO 1558-1560; 1562-1564; 1566; 1572; 1576.

Progoniada regularis Hartman, 1965

Progoniada regularis Hartman, 1965: 100-101, pl. 16

Progoniada regularis - Rizzo, 2002: 97, figs. 39-41, tables. 8-9 ; Rizzo and Amaral, 2004a: 48, figs. 13, table 1; Amaral et al., 2004: 202, appendix 3; Böggemann, 2005: 155, figs. 86-88.

\section{Hesionidae Grube, 1850}

Hesionides Friedrich, 1937

Hesionides sp.

Deposit number - MCEM - BPO1472 .

Ophiodromus obscurus (Verrill, 1873)

Podarke obscura Verrill, 1873: 589, fig. 61.

Podarke obscura — Lana, 1984: 57; 1987: 1061, table 1.

Deposit number - MCEM - BPO 1442. 


\section{LISTS OF SPECIES}

Parahesione Pettibone, 1956

Parahesione sp.

Obs: Identified in an unpublished thesis (Lorenzi, 1998). Identification confirmed by Fredrik Pleijel, but material subsequently lost.

\section{Lumbrineridae Schmarda, 1861}

Arabelloneris janeirensis (Augener, 1934)

Lumbriconeris janeirensis Augener, 1934: 198, fig. 28a-c.

Lumbrineris janeirensis — Lana, 1984:171, figs. 188-189; 1987: 1061, table 1.

Arabelloneris janeirensis - Camargo, 1993: 36, fig. 6a-c; Camargo and Lana, 1995a: 81, figs. 7-9, 29.

Deposit number-MCEM - BPO 789; 846.

Lumbricalus januarii (Grube, 1878)

Lumbriconereis januarii Grube, 1878: 91.

Lumbrineris januarii - Lana, 1984: 172, figs. 190-192; 1987: 1061, table 1; Camargo and Lana, 1995b:

103, figs. 22-27, 60 .

Lumbricalus januarii - Carrera-Parra, 2004:84, figs. 3A-G.

Deposit number - MCEM - BPO 745; 755; 860; 867; 870.

Lumbrineris atlantica (Kinberg, 1865)

Lumbriconeris atlantica Kinberg, 1865: 568.

Lumbrineris atlantica Camargo and Lana, 1995b: 95, figs. 1-5, 60.

Deposit number - MCEM - BPO 821; 866.

Lumbrineris cingulata (Ehlers, 1897)

Lumbriconeris cingulata Ehlers, 1897: 76, figs. 119-124.

Lumbrineris cingulata - Lana, 1984: 177, fig. 194a-b; 1987: 1061, table 1; Camargo and Lana, 1995b: 112, figs. 49-54, 61.

Deposit number - MCEM - BPO 773; 788; 798; 800; 802-805; 807; 815; 817; 824; 825; 831; 835-842.

Lumbrineris curtolobata Camargo and Lana, 1995b

Lumbrineris curtolobata Camargo and Lana, 1995b: 1001, figs. 18-21, 60.

Lumbrineris hebes Verrill, 1880

Lumbrineris hebes Verrill, 1880: 174.

Lumbrineris hebes - Lana, 1987: 1061, table 1.

Lumbrineris inflata Moore, 1911

Lumbrineris inflata Moore, 1911: 289, figs. 128-134.

Lumbrineris inflata — Lana, 1984: 175; 1987: 1061, table 1; Camargo, 1993: 76, fig. 15a-f; Camargo and Lana, 1995b: 104, figs. 28-33, 60.

Deposit number - MCEM - BPO 736 ; $775 ; 783-785 ; 792-794$.

Lumbrineris magalhaensis Kinberg, 1865

Lumbrineris magalhaensis Kinberg, 1865: 568.

Lumbrineris magalhaensis - Camargo, 1993: 90, fig. 19a-f; Camargo and Lana, 1995b: 110, figs. 4548,61 .

Deposit number - MCEM - BPO 771; 772; 804; 811; 814; 818; 821; 826; 827; 850; 851; 853; 858; 865.

Lumbrineris latreilli Audouin and Milne Edwards, 1834

Lumbrineris latreilli Audouin and Milne Edwards, 1834: 168.

Lumbrineris latreilli - Lana, 1987: 1061, table 1; Camargo, 1993: 100, fig. 21a-e; Camargo and Lana, 1995b: 115, figs. 55-59, 61 .

Deposit number - MCEM - BPO 768; 770; 790; 854.

Lumbrineriopsis mucronata (Ehlers, 1908)

Lumbriconeris mucronata Ehlers, 1908: 95, figs. 9-13.

Lumbrineriopsis mucronata - Lana, 1984: 170, figs. 186-187; 1987: 1061, table 1; Camargo, 1993: 40, fig. 7a-c; Camargo and Lana, 1995a: 82, figs. 10-14, 29. 


\section{LISTS OF SPECIES}

Lysarete brasiliensis Kinberg, 1865

Lysarete brasiliensis Kinberg, 1865: 570.

Lysarete brasiliensis — Lana, 1984: 197, fig. 214; 1987: 1061, table 1; Camargo and Lana, 1995a: 79, figs. 1-6, 29.

Deposit number - MCEM - BPO 799; 874.

Ninoe brasiliensis Kinberg, 1865

Ninoe brasiliensis Kinberg, 1865: 567.

Ninoe brasiliensis — Lana, 1984: 168, fig. 185; 1987: 1061, table 1; Camargo, 1993: 111, fig. 24a-d;

Camargo and Lana, 1995a: 87, figs. 25-28, 29.

Deposit number - MCEM - BPO 737-743; 777-781; 813; 830-834; 856; 859; 861.

Scoletoma tetraura (Schmarda, 1861)

Notocirrus tetraurus Schmarda, 1861: 117.

Scoletoma tetraura - Camargo, 1993: 62, fig. 12a-f; Camargo and Lana, 1995b: 99, figs. 12-17, 60; Amaral and Nallin, 2005: 48, table 2.

Deposit number - MCEM - BPO 752.

\section{Magelonidae Cunningham and Ramage, 1888}

Magelona crenulata Bolívar and Lana, 1986

Magelona crenulata Bolívar and Lana, 1986: 144, figs. 43-49.

Deposit number - MCEM - BPO 200-201.

Magelona papillicornis Müller, 1858

Magelona papillicornis Müller, 1858: 215, figs. 10-11.

Magelona papillicornis - Bolívar, 1986: 46, figs. 102-105; Bolívar and Lana, 1986: 136, figs. 9-12.

Deposit number - MCEM - BPO 99-107; 176-180; MZUSP 128.

Magelona posterelongata Bolívar and Lana, 1986

Magelona posterelongata Bolívar and Lana, 1986: 140, figs. 22-30.

Magelona posterelongata - Bolívar, 1986: 51, figs. 115-123.

Deposit number - MCEM - BPO 126-129; MZUSP 129.

Magelona riojai Jones, 1963

Magelona riojai Jones, 1963: 9, figs. 22-35.

Magelona riojai - Bolívar, 1986: 44, figs. 94-101; Bolívar and Lana, 1986: 135, figs. 1-8; Amaral and Nallin, 2005: 48, table 2.

Deposit number - MCEM - BPO 97-98; 175.

Magelona variolamellata Bolívar and Lana, 1986

Magelona variolamellata Bolívar and Lana, 1986:137, figs. 13-28.

Magelona variolamellata - Bolívar, 1986: 47, figs. 106-114; Blankensteyn and Moura, 2002: 718, table 2.

Deposit number - MCEM - BPO 108-125; 181-195; MZUSP 130-132.

\section{Maldanidae Malmgren, 1867}

Asychis amphiglypta (Ehlers, 1897)

Maldane amphiplypta Ehlers, 1897: 119, Pl. 8, figs. 187-193.

Asychis amphiglypta — Bolívar, 1990: 86, fig. 66a-e.

Deposit number - MCEM - BPO 687.

Axiothella brasiliensis (Mangum, 1966)

Clymenella brasiliensis Mangum, 1966: 5, fig. 1a-d.

Axiothella brasiliensis - Bolívar, 1990: 70, fig. 51a-f.

Deposit number - MCEM - BPO 595-597; 600-602; 604; 606; 607; 609-611.

Axiothella sp.

Deposit number - MZUSP 318. 


\section{LISTS OF SPECIES}

\section{Chirimia amoena (Kinberg, 1867)}

Chrysothemis amoena Kinberg, 1867: 340.

Chirimia amoena - Bolívar, 1990: 88, fig. 68a-f.

Deposit number - MCEM - BPO 699; 702; 704; 705.

Clymenela dalesi Mangum, 1966

Clymenella dalesi Mangum, 1966: 1, fig. 2a-d.

Clymenella dalesi - Bolívar, 1990: 74, fig. 2a-d.

Deposit number - MCEM - BPO 631; 634; 638; 641; 644; 645; 647.

Rhodine loveni Malmgren, 1865

Rhodine loveni Malmgren, 1865: 189.

Rhodine loveni - Bolívar, 1990: 79, fig. 59a-e.

Deposit number - MCEM - BPO 655-657.

Obs: This is probably a misidentification and this material needs to be revised.

\section{Nephtyidae Grube, 1850}

Aglaophamus juvenalis Kinberg, 1866

Aglaopheme juvenalis Kinberg, 1866: 240.

Aglaophamus juvenalis - Lana, 1984: 86, figs. 78-79; 1986: 143, figs. 16-17; 1987: 1061, table 1.

Deposit number - MCEM - BPO 26-31; 218; 218.

Aglaophamus uruguayi Hartman, 1953

Aglaophamus uruguayi Hartman, 1953: 32, fig. 8a-d.

Aglaophamus uruguayi - Lana, 1984: 77, figs. 68-69; 1986: 144, figs. 18-19; 1987: 1061, table 1.

Deposit number - MCEM - BPO 32-34; 220-228.

Inermonephtys palpata Paxton, 1974

Inermonephtys palpata Paxton, 1974: 200, figs. 2-6.

Inermonephtys palpata - Lana, 1984: 84, figs. 72-77; 1986: 141, figs. 10-15; 1987: 1061, table 1.

Deposit number - MCEM - BPO 23-25.

Obs: This is a new species, currently being reviewed by Martin, Gil and Lana.

Nephtys acrochaeta Hartman, 1950

Nephtys acrochaeta Hartman, 1950: 114, figs. 1-6.

Nephtys acrochaeta - Lana, 1984: 77, figs. 63-65; 1986: 138, figs. 1-3; 1987: 1061, table 1.

Deposit number - MCEM - BPO 1-5.

Nephtys fluviatilis Monro, 1937

Nephtys fluviatilis Monro, 1937: 246, fig. 2a-c.

Nephtys fluviatilis — Lana, 1984: 77, figs. 68-69; 1986: 140, figs. 6-7; 1987: 1061, table 1; Blankensteyn and Moura, 2002: 718, table 2.

Deposit number - MCEM - BPO 13-16; 216; 217.

Nephtys simoni Perkins, 1980

Nephtys simoni Perkins, 1980: 37, figs. 15-16.

Nephtys simoni - Lana, 1984: 82, figs. 70-71; 1986: 141, figs. 8-9; 1987: 1061, table 1; Amaral and Nallin 2005: 48, table 2.

Deposit number - MCEM - BPO 17-22; 216; 217.

Nephtys squamosa Ehlers, 1887

Nephtys squamosa Ehlers, 1887: 128, figs. 7-10.

Nephtys squamosa — Lana, 1984: 77, figs. 66-67; 1986: 139, figs. 4-5; 1987: 1061, table 1.

Deposit number - MCEM - BPO 6-12; 202-212. 


\section{LISTS OF SPECIES}

\section{Nereididae Johnston, 1865}

Ceratocephale loveni Malmgren, 1867

Ceratocecephale loveni Malmgren, 1867: 61-62.

Ceratocephale loveni - Attolini, 2002: 25, figs. 8, 9, 11, 12, tables 4, 8.

Ceratonereis excisa (Grube, 1874)

Nereis excisa Grube, 1874: 72-73.

Ceratonereis excisa - Lana, 1987: 1061, table 1.

Deposit number - MCEM - BPO 1132-1138; 1375-1376.

Gymnonereis crosslandi (Banse, 1977)

Ceratocephale crosslandi Banse, 1977: 620, fig. 3.

Ceratocephale oculata Banse, 1977

Ceratocephale oculata — Lana, 1984: 113, figs. 109-111.

Deposit number - MCEM - BPO 963-968; 970-983; 985-987; 989-1005.

Laeonereis acuta (Treadwell, 1923)

Nereis acuta Treadwell, 1923: 3, figs. 1-5.

Laeonereis acuta - Lana, 1984: 115, figs. 112-113; 1987: 1061, table 1; Blankensteyn and Moura, 2002: 718, table 2; Amaral and Nallin, 2005: 48, table 2.

Deposit number - MCEM - BPO 1155-1209; 1369-1372; 1374.

Micronereides capensis Day, 1963

Micronereides capensis Day, 1963: 404, fig. 6a-e.

Micronereides capensis - Amaral et al., 2004: 202, appendix 3; Amaral et al., 2006: 122.

Deposit number -MCEM - BPO 1271-1277; 1280; 1283; 1289.

Namalycastis abiuma (Müller in Grube, 1872)

Paranereis abiuma Müller in Grube, 1872: 47.

Namalycastis abiuma — Lana, 1984: 109, figs. 105-106; 1987: 1061, table 1; Glasby, 1999: 31, figs. 1c, 8a-b, 9 .

Deposit number - MCEM -BPO 876; 1216; 1341.

Namanereis amboinensis (Pflugfelder, 1933)

Lycastopsis amboinensis Pflugfelder, 1933: 69-71, figs. 9-11.

Namanereis amboinensis - Hartman. 1959: 162-163; Glasby, 1999: 76, figs. 1c, 3, 31 a-g.

Lycastopsis pontica — Lana, 1987: 1061, table 1; Amaral and Nallin, 2005: 48, table 2.

Neanthes bruaca Lana and Sovierzoski, 1987

Neanthes bruaca Lana and Sovierzoski, 1987: 678, figs. 1-4.

Neanthes bruaca - Lana, 1984: 133, figs. 128-134a-c; 1987: 1061, table 1; Steiner and Santos, 2004: 39, figs. 1-6.

Deposit number - MCEM - BPO 233; 235-239; 1222-1225; 1295-1309; 1400-1409; 1413; MZUSP 140.

Neanthes ceciliae Steiner and Santos, 2004

Neanthes ceciliae Steiner and Santos, 2004: 47, figs. 7-14, table1.

Neanthes succinea (Frey and Leuckart, 1847)

Nereis succinea Frey and Leuckart, 1847: 154.

Neanthes succinea - Lana, 1984: 136, figs. 135; 1987: 1061, table 1; Blankensteyn and Moura, 2002: 718 , table 2.

Deposit number - MCEM - BPO 1015-1030; 1345-1357; 1411; 1412; MZUSP 140.

Nereis broa Lana and Sovierzoski, 1987

Nereis broa Lana and Sovierzoski, 1987: 683, figs. 5-8.

Nereis broa - Amaral et al., 2006: 127.

Deposit number - MCEM -BPO 240.

Nereis oligohalina (Rioja, 1946)

Neanthes oligohalina Rioja, 1946: 207, figs. 3-6 and 13-19. 


\section{LISTS OF SPECIES}

Nereis oligohalina - Lana, 1984: 131, figs. 127a-b; 1987: 1061, table 1; Blankensteyn and Moura, 2002: 718, table 2; Amaral and Nallin, 2005: 48, table 2.

Deposit number - MCEM - BPO 1090-1100; 1102; 1103; 1337-1340; 1633-1634.

Obs: This is probably a misidentification and this material needs to be revised.

Nereis riisei Grube, 1857

Nereis riisei Grube, 1857: 162.

Nereis riisei — Lana, 1984: 128, figs. 124-124; 1987: 1061, table 1.

Deposit number - MCEM - BPO 1037-1089; 1101; 1342-1344.

Nereis zonata Malmgren, 1867

Nereis zonata Malmgren, 1867: 164, fig. 34.

Nereis zonata - Lana, 1984: 130, figs. 126a-b; 1987: 1061, table 1.

Deposit number - MCEM - BPO 1334-1335.

Nereis cf. persica Fauvel 1911

Nereis cf. persica Fauve1, 1911; 385.

Deposit number - MCEM - BPO 1389-1398; 1393.

Perinereis anderssoni Kinberg, 1866

Perinereis anderssoni Kinberg, 1866: 175.

Perinereis anderssoni - Lana, 1984: 126, figs. 122-123; 1987: 1061, table 1.

Deposit number - MCEM - BPO 896-905;1386-1388.

Perinereis cultrifera (Grube, 1840)

Nereis cultrifera Grube, 1840: 74.

Perinereis cultrifera - Nonato, 1981: 96, figs. 99-106.

Deposit number-MCEM -BPO 886-889.

Perinereis ponteni Kinberg, 1866

Perinereis ponteni Kinberg, 1866: 176.

Perinereis ponteni - Lana, 1984: 123, figs. 119-121; 1987: 1061, table 1.

Deposit number - MCEM -BPO 906-934; 1358-1365.

Perinereis vancaurica (Ehlers, 1868)

Nereis vancaurica Ehlers, 1868: 20.

Perinereis vancaurica - Lana, 1984: 121, figs. 118; 1987: 1061, table 1; Blankensteyn and Moura, 2002: 718, table 2 .

Deposit number - MCEM - BPO 890-894; 1378-1385.

Obs: This is probably a misidentification and this material needs to be revised.

Platynereis dumerilii (Audouin and Milne-Edwards, 1833)

Nereis dumerilii Audouin and Milne-Edwards, 1833: 154, fig. 43.

Platynereis dumerilii - Lana, 1984: 117, figs. 114a-b; 1987: 1061, table 1; Amaral and Nallin, 2005: 48, table 2.

Deposit number - MCEM - BPO 936-962; 1377; 1414; 1418; MZUSP 154.

Pseudonereis gallapagensis Kinberg, 1866

Pseudonereis gallapagensis Kinberg, 1866: 174.

Pseudonereis gallapagensis - Lana, 1984: 118, figs. 115-117; 1987: 1061, table 1; Amaral and Nallin, 2005: 48, table 2.

Deposit number - MCEM - BPO 882-885; 1367; 1368.

\section{Oenonidae Kinberg, 1865}

Arabella iricolor (Montagu, 1804)

Neris iricolor Montagu, 1804: 82.

Arabella iricolor - Lana, 1984: 192, figs. 208-209; 1987: 1061, table 1; Amaral and Nallin, 2005: 48, table 2 .

Drilonereis filum (Claparede, 1870) 


\section{LISTS OF SPECIES}

Lumbriconereis filum Claparede, 1870: 454, fig. 1.

Drilonereis filum - Lana, 1984: 193, figs. 210-211; 1987: 1061, table 1; Amaral and Nallin, 2005: 48, table 2

Notocirrus lorum Ehlers, 1897

Notocirrus lorum Ehlers, 1897: 78.

Notocirrus lorum — Lana, 1987: 1061, table 1.

\section{Onuphidae Kinberg, 1865}

Australonuphis casamiquelorum (Orensanz, 1974)

Americonuphis casamiquelorum Orensanz, 1974: 100, pl. 9, figs. 1-14.

Australonuphis casamiquelorum — Lana, 1987: 1061, table 1; 1991: 286; Amaral and Nallin, 2005: 48, table 2.

Deposit number - MCEM - BPO 429-430.

Brevibrachium hannelorae Lana, 1991

Rhamphobrachium cf. agassizii — Lana, 1984: 148, table 1; 1987: 1061, table 1.

Brevibrachium hannelorae Lana, 1991: 284, figs. 2 a-m.

Deposit number - MCEM - BPO 367; 368.

Diopatra dexiognatha Paxton and Bailley-Brock, 1986

Diopatra dexiognatha Paxton and Bailley-Brock, 1986: 1-4, figs. 1-16. - Steiner, 2006: 101, figs. 35-39.

Diopatra cuprea — Lana, 1984: 142, figs. 138-140; 1987: 1061, table 1.

Diopatra viridis - Lana, 1991: 283; Amaral and Nallin, 2005: 48, table 2.

Deposit number - MCEM - BPO 371-377.

Diopatra tridentata Hartman, 1944

Diopatra tridentata Hartman, 1944: 61, figs, 37-43.

Diopatra tridentata - Lana, 1984: 144, figs. 141-142; 1987: 1061, table 1; 1991: 283; Amaral et al., 2004: 203, appendix 3.

Deposit number - MCEM - BPO 379-393.

Hyalinoecia juvenalis Moore, 1911

Hyalinoecia juvenalis Moore, 1911: 277, figs. 86-95.

Hyalinoecia juvenalis - Nonato, 1981: 117; Lana, 1984: 140, figs. 136-137; 1987: 1061, table 1; 1991: 283.

Kinbergonuphis atlantisa (Hartman, 1965)

Nereis atlantisa Hartman, 1965: 102-104, figs. 17a-c.

Kinbergonuphis atlantisa - Attolini, 2002: 25, figs. 8, 9, 11, 12, tables 4, 8 .

Kinbergonuphis difficilis (Fauchald, 1982)

Onuphis difficilis Fauchald, 1982: 203, fig. 1.

Kinbergonuphis difficilis — Lana, 1984 : 165, fig. 184; 1987: 1061, table 1; 1991: 290.

Deposit number - MCEM - BPO 424-426; 428.

Kinbergonuphis fauchaldi Lana, 1991

Kinbergonuphis fauchaldi - Lana, 1984: 161, figs. 177-181 (Original name in an upublished thesis)

Kinbergonuphis fauchaldi Lana, 1991: 286, fig. 3a-k;

Deposit number - MCEM - BPO 413- 414; 417.

Kinbergonuphis nonatoi Lana, 1991

Kinbergonuphis nonatoi - Lana, 1984: 159, figs 170-176 (Original name in an unpublished thesis)

Kinbergonuphis nonatoi Lana, 1991, table 1; 1991: 288, figs. 4a-1.

Deposit number - MCEM - BPO 411; 412.

Kinbergonuphis orensanzi (Fauchald, 1982)

Onuphis orensanzi Fauchald, 1982: 205, fig. 2.

Kinbergonuphis orensanzi — Lana, 1984: 163, figs. 183; 1987: 1061, table 1; 1991: 290.

Deposit number - MCEM - BPO 415; 416; 418; 419; 421-423; 1469. 


\section{LISTS OF SPECIES}

Mooreonuphis lineata Lana, 1991

Mooreonuphis lineata - Lana, 1984: 152, figs. 157-164 (Original name in an upublished thesis)

Mooreonuphis lineata — Lana, 1991: 291, figs. 5a-m.

Deposit number - MCEM - BPO 395-400; 434-435; MZUSP 139.

Mooreonuphis intermedia (Kinberg, 1865)

Onuphis intermedia Kinberg, 1865: 560.

Mooreonuphis intermedia - Lana, 1984: 155, figs 165-168.

Deposit number - MCEM - BPO 402-405; 407-409.

Mooreonuphis pallidula (Hartman, 1965)

Nothria pallidula Hartman, 1965: 1965, figs. d-h, pl. 17.

Mooreonuphis pallidula - Amaral et al., 2006: 203.

Nothria benthophyla Lana, 1991

Nothria benthophyla - Temperini, 1981: 32, figs. 62-72 (Original name in an upublished thesis).

Nothria benthophyla Lana, 1984: 150, figs. 146-156; 1987: 1061, table 1.

Nothria benthophyla Lana, 1991: 280, figs. 1a-m.

Deposit number - MCEM - BPO 394.

Onuphis eremita oculata Hartman, 1951

Onuphis eremita oculata Hartman, 1951: 52, figs. 1-2.

Onuphis eremita oculata - Lana, 1984: 158, fig. 169; 1991: 293.

Deposit number -MCEM - BPO 410

Rhamphobrachium verngreni (Kinberg, 1865)

Onuphis verngreni Kinberg, 1865: 560.

Ramphobrachium verngreni - Lana, 1984: 146, figs. 143-144; 1987: 1061, table 1; 1991: 286.

\section{Opheliidae Malmgren 1867}

Armandia hossfeldi Hartmann-Schröder, 1956

Armandia hossfeldi Hartmann-Schröder, 1956: 67, figs. 10-11

Armandia hossfeldi - Elías et al., 2003: 78.

Deposit number - MCEM - BPO 1584-1588.

Armandia maculata (Webster, 1884)

Ophelina maculata Webster, 1884: 322, pl. 11, figs. 54-55.

Armandia maculata - Elías et al., 2003: 79.

Armandia loboi Elías and Bremec, 2003

Armandia loboi Elías and Bremec, 2003: 181.

Deposit number - MCEM - BPO 1590; 1591.

Euzonus furciferus (Ehlers, 1897)

Thoracophelia furcifera Ehlers, 1897: 101

Euzonus furciferus - Blankensteyn and Moura, 2002: 718, table 2; Amaral and Nallin, 2005: 48, table 2.

Obs: This is probably a misidentification and this material needs to be revised.

Ophelina acuminata Oersted, 1843

Ophelina acuminata Oersted, 1843: 2, P1. 46.

Ophelina cf. acuminata - Elías et al., 2003: 81.

Deposit number-MCEM - BPO 1592-1606; 1612.

Ophelina alata Elías, Bremec, Lana and Orensanz, 2003

Ophelina alata Elías, Bremec, Lana and Orensanz, 2003: 83, fig. 3.

Deposit number - MCEM - BPO 1607-1609; 1613-1616.

Ophelina cylindricaudata (Hansen, 1878)

Ammotrypane cylindricaudata Hansen, 1878: 8

Ophelina cylindricaudata - Amaral et al., 2004: 201, appendix 3. 


\section{LISTS OF SPECIES}

Ophelina gaucha Elías, Bremec, Lana and Orensanz, 2003

Ophelina gaucha Elías, Bremec, Lana and Orensanz, 2003: 82, fig. 2.

Deposit number - MCEM - BPO 1610.

Travisia sp.

Deposit number - MCEM - BPO 1475.

\section{Orbiniidae Hartman, 1942}

Leitoscoplos kerguelensis (McIntosh, 1885)

Scoloplos kerguelensis Mclntosh, 1885: Pl. 43, figs. 6-8, pl. 221, fig. 19.

Leitoscoplos kerguelensis - Bolívar, 1990: 27, fig. 19a-e.

Leitoscoloplos robustus (Verrill, 1873) Anthostoma robustum Verrill, 1873: 597, Pl. 14, fig. 76.

Leitoscoloplos robustus - Bolívar, 1990: 26, fig. 18a-d; Amaral and Nallin, 2005: 48, table 2.

Naineris setosa (Verrill, 1900)

Aricia setosa Verrill, 1990: 651.

Naineris setosa - Bolívar, 1990: 11, fig. 5a-d.

Deposit number - MCEM - BPO 436; 731-732.

Orbinia latreillii (Audouin and Milne-Edwards, 1834)

Aricia latreillii Audouin and Milne-Edwards, 1834: 259.

Orbinia cf. latreillii - Bolívar, 1990: 23, fig. 15a-e.

Deposit number - MCEM - BPO 479-480.

Phylo felix Kinberg, 1866

Phylo felix Kinberg, 1866: 251.

Phylo felix - Bolívar, 1990: 12, fig. 6a-e.

Deposit number - MCEM - BPO 437-440.

Scoloplos capensis (Day,1961)

Scolaricia capensis Day, 1961: 480, pl. 17, fig. 1p-s.

Scoloplos capensis - Bolívar, 1990: 19, fig. 12a-d.

Deposit number - MCEM - BPO 471; 472.

Scoloplos ohlini (Ehlers, 1901)

Aricia ohlini Ehlers, 1901: 167, P1. 21, figs. 9-13.

Scoloplos ohlini - Bolívar, 1990: 14, fig. 8a-d.

Deposit number - MCEM - BPO 442-457; MZUSP 263.

Scoloplos rubra (Webster, 1879)

Aricia rubra Webster, 1879: 253, Pl. 9, figs. 123-126.

Scoloplos rubra - Bolívar, 1990: 16, fig. 9a-e.

Deposit number - MCEM - BPO 458-461.

Scoloplos scoloplos Blainville, 1828

Scoloplos scoloplos Blainville, 1828: 628

Scoloplos treadwelli Eisig, 1914

Scoloplos treadwelli Eisig, 1914: 21 figs. 161-169.

Scoloplos treadwelli - Attolini, 2002: 26, figs. 8, 9, 11, 12, tables 4, 8.

Scoloplos verax (Kinberg, 1866)

Leodamas verax Kinberg, 1866: 252.

Scoloplos verax - Bolívar, 1990: 17, fig. 10a-c.

Deposit number - MCEM - BPO 462-466; 468.

\section{Oweniidae delle Chiaje, 1844}

Galathowenia Kirkegaard, 1959

Galathowenia sp. 


\section{LISTS OF SPECIES}

Obs: Identified in an unpublished thesis (Lorenzi, 2004), but material subsequently lost.

Owenia brasiliensis Hansen, 1882

Amochares brasiliensis Hansen, 1882: 19, Pl. 5, fig. 33-36, Pl. 6, figs. 1-4.

Owenia sp. 1 - Sene-Silva, 2003: 79.

Owenia fusiformis delle Chiaje, 1844

Owenia fusiformis delle Chiaje 1844: 31.

Owenia fusiformis - Nonato, 1981: 195; Amaral and Nallin, 2005: 48, table 2.

Obs: This is probably a misidentification and this material needs to be revised.

\section{Paralacydoniidae Pettibone, 1963}

Paralacydonia paradoxa Fauvel, 1913

Paralacydonia paradoxa Fauvel, 1913: 54, fig. 10.

Paralacydonia paradoxa - Rizzo, 2002:117, figs. 42, 43.

\section{Paraonidae Cerruti, 1909}

Aparaonis sp.

Deposit number - MZUSP 56.

Aricidea albatrossae Pettibone, 1957

Aricidea albatrossae Pettibone, 1957: 354, fig. 1a-f.

Aricidea cf. albatrossae - Bolívar, 1990: 30, fig. 20a-d.

Aricidea fragilis Webster, 1879

Aricidea fragilis Webster, 1879: 55, pl. 9, figs. 127-132.

Aricidea cf. fragilis - Bolívar, 1990: 31, fig. 21a-d.

Deposit number - MCEM - BPO 495.

Aricidea taylori Pettibone, 1965

Aricidea taylori Pettibone, 1965: 131, figs. 4a-c, 5a-c.

Aricidea taylori - Bolívar, 1990: 34, fig. 24a-e.

Aricidea simplex (Day, 1963)

Aricidea suecica simplex Day, 1963: 364, fig. 3a-b.

Aricidea simplex - Bolívar, 1990: 37, fig. 26a-f.

Cirrophorus branchiatus Ehlers, 1908

Cirrophorus branchiatus Ehlers, 1908: 124, P1. 17, figs. 5-9.

Cirrophorus branchiatus - Bolívar, 1990: 38, fig. 27a-e.

Deposit number - MCEM - BPO 509-511.

Paraonis pygoenigmatica Jones, 1968

Paraonis pygoenigmatica Jones, 1968: 323, figs. 1-23.

Paraonis pygoenigmatica - Amaral and Nallin, 2005: 48, table 2.

\section{Pectinariidae de Quatrefages, 1866}

Pectinaria cf. catarinensis Grube, 1871

Pectinaria catarinensis Grube, 1871: 72.

Deposit number - MZUSP152.

\section{Pilargidae Saint-Joseph, 1899}

Ancistrosyllis jonesi Pettibone, 1966

Ancistrosyllis jonesi Pettibone, 1966: 173, figs. 9-10.

Ancistrosyllis jonesi — Lana, 1984: 61; 1987: 1061, table 1. 


\section{LISTS OF SPECIES}

Deposit number - MCEM - BPO 1490.

Litocorsa Pearson, 1970

Litocorsa sp.

Obs: Identified in an unpublished thesis (Lorenzi, 2004), but material subsequently lost.

Parandalia tricuspis (Müller, 1858)

Loandalia americana Hartman, 1947: 506, figs. 1-7.

Parandalia americana - Lana, 1984: 59; 1987: 1061, table 1; Blankensteyn and Moura, ,2002: 718, table 2.

Parandalia tricuspis - Salazar-Vallejo, 1990: 512, figs. 3, 4d-f.

Deposit number - MCEM - BPO 1485-1487.

Sigambra grubei Müller, 1858

Sigambra grubei Müller, 1858: 214, figs. 7-9.

Sigambra grubii — Nonato, 1981: 90, figs. 97-98; Lana, 1984: 61; 1987: 1061, table 1; Amaral et al., 2006: 48, table 2.

Deposit number - MCEM - BPO 1488.

Sigambra sp.

Deposit number - MCEM - BPO 1489.

\section{Pisionidae Ehlers, 1901}

Pisionidens indica Aiyar and Alikunhi, 1940

Pisionidens indica Aiyar and Alikunhi, 1940: 106.

Pisionidens indica - Amaral et al., 2006: 120; Amaral and Nallin, 2005: 48, table 2.

Deposit number - MCEM - BPO 1228; 1478.

Pisione Grube, 1857

Pisione sp.

Deposit number - MCEM - BPO 1477.

\section{Phyllodocidae Oersted, 1843}

Anaitides tamoya Nonato, 1981 nomen nudum. Anaitides tamoya Nonato, 1981: 77. (Original name in an unpublished thesis)

Anaitides tamoya - Lana, 1987: 1061, table 1.

Eulalia myriacicla (Schmarda, 1861)

Notophyllum myriacicla Schmarda, 1861: 87, fig. 233.

Eulalia myriacicla — Lana, 1984: 53; 1987: 1061, table 1.

Deposit number - MCEM - BPO 1484.

Genetyllis castanea (Marenzeller, 1879)

Carobia castanea Marenzeller, 1879: 127, fig. 2.

Genetyllis castanea - Lana, 1984: 54; 1987: 1061, table 1.

Deposit number - MCEM - BPO 1483.

Hesionura Hartmann-Schröder, 1958

Hesionura sp.

Deposit number - MCEM - BPO 1471.

Phyllodoce mucosa Oersted, 1843

Phyllodoce mucosa Oersted, 1843: 31.

Phyllodoce cf. mucosa - Lana, 1984: 56. 


\section{LISTS OF SPECIES}

\section{Pholoididae Kinberg, 1858}

Pholoe minuta (Fabricius, 1780) Aphrodita minuta Fabricius, 1780: 414.

Pholoe minuta - Lana, 1984: 33; 1987: 1061, table 1; Amaral and Nonato, 1984: 17, figs. 19-25. Obs: This is probably a misidentification and this material needs to be revised.

\section{Polygordiidae Czerniavsky, 1881}

Polygordius Schneider, 1868

Polygordius sp.

Deposit number - MCEM - BPO 1474.

\section{Polynoidae Malmgren, 1867}

Eunoe papilosa Amaral and Nonato, 1982

Eunoe papilosa Amaral and Nonato, 1982: 19, figs. 32-37.

Eunoe papilosa - Lana, 1987: 1061, table 1.

Eunoe serrata Amaral and Nonato, 1982

Eunoe serrata Amaral and Nonato, 1982: 19, figs. 38-44.

Eunoe serrata - Lana, 1984: 22; 1987: 1061, table 1.

Deposit number - MCEM - BPO 1230; 1231.

Halosydnella brasiliensis (Kinberg, 1858)

Halosydna brasiliensis Kinberg, 1858: 16, fig. 22.

Halosydnella brasiliensis — Lana, 1984: 21; 1987: 1061, table 1.

Deposit number - MCEM - BPO 1232.

Harmothoe lunulata (delle Chiaje, 1841)

Polynoe lunulata delle Chiaje, 1841 in Claparède, 1868: 373.

Harmothoe lunulata — Lana, 1984: 23; 1987: 1061, table 1.

Lepidasthenia virens (Blanchard, 1849)

Polynoe virens Blanchard, 1849: 16.

Lephidasthenia virens - Lana, 1987: 1061, table 1.

Subadyte pellucida (Ehlers, 1864)

Polynoe pellucida Ehlers, 1864: 105, fig. 10, est. 3, figs 5, 7-13, est. 4, figs 1-3.

Subadyte pellucida - Lana, 1984: 19; 1987: 1061, table 1.

Deposit number - MCEM - BPO 1229.

\section{Protodrilidae Czerniavsky, 1881}

Protodrilus Hatschek, 1880

Protodrilus sp.

Deposit number - MCEM - BPO 1473

\section{Sabellariidae Johnston, 1865}

Phragmatopoma caudata Mörch, 1963

Phragmatopoma lapidosa Kinberg, 1867: 349.

Phragmatopoma caudata - Kirtley 1994: 31, figs. 2.3.1-2.3.6.

Phragmatopoma lapidosa - Lana and Bremec, 1994: 21.

Deposit number - MZUSP 153. 


\section{LISTS OF SPECIES}

Sabellaria bella Grube, 1870

Sabellaria bella Grube, 1870: 69.

Sabellaria bella - Gruet and Lana, 1998: 32, figs. 1-2; Lana and Gruet, 1989: 243; Lana and Bremec, 1994: 21; Kirtley, 1994: 53, fig. 4.3

Deposit number - MCEM - BPO 251.

Sabellaria bellis Hansen, 1882

Sabellaria bellis Hansen, 1882: 19, pl. VI, figs. 5-17.

Sabellaria bellis — Rullier and Amoureux, 1979: 188; Gruet and Lana, 1998: 34, figs. 3-4; Kirtley 1994: 54, fig. 4.5.1-4.5.2.

Sabellaria wilsoni Lana and Gruet, 1989

Sabellaria wilsoni Lana and Gruet, 1989: 239, figs. 1-21.

Sabellaria wilsoni - Kirtley, 1994: 80, fig. 4.33.

Deposit number - MCEM - BPO 241-244; 431; 730.

\section{Sabellidae Malmgren, 1867}

Potamilla fonticula (Hoagland, 1919)

Parasabella fonticula Hoagland, 1919: 579.

Potamilla fonticula — Rullier and Amoureux, 1979: 191.

\section{Saccocirridae Czerniavsky, 1881}

Saccocirrus Bobretzky, 1871

Saccocirrus sp.

Deposit number - MCEM - BPO 1479.

\section{Scalibragmatidae Malmgren 1867}

Scalibregma inflatum Rathke, 1843

Scalibregma inflatum Rathke, 1843: 184, Pl. 9, figs. 15-21

Scalibregma inflatum — Bolívar, 1990: 90, fig. 69a-d.

\section{Sigalionidae Kinberg, 1856}

Ehlersileanira incisa (Grube, 1878)

Sthenelais incisa Grube, 1878: 519.

Ehlersileanira incisa — Lana, 1984: 46; 1987: 1061, table 1; 1991: 131.

Deposit number - MCEM- BPO 172-174.

Fimbriosthenelais marianae Lana, 1991

Fimbriosthenelais marianae - Lana, 1984: 39; 1987: 1061, table 1 (Original name in an upublished thesis ).

Fimbriosthenelais marianae Lana, 1991: 128, figs. 22-35.

Deposit number - MCEM - BPO 145-147

Psammolyce catenulata Amaral and Nonato, 1984

Psammolyce catenulata - Amaral and Nonato, 1984: 20, figs. 33-43; Lana, 1987: 1061, table 1.

Psammolyce catenulata — Lana, 1984: 34; 1991: 124.

Psammolyce flava Kinberg, 1855

Psammolyce flava Kinberg, 1855: 388.

Psammolyce flava - Lana, 1991: 125; 1987: 1061, table 1.

Sigalion arenicola Verrill, 1879

Sigalion arenicola Verrill, 1879: 167. 


\section{LISTS OF SPECIES}

Sigalion arenicola — Lana, 1984 : 126, figs. 13-16; 1987: 1061, table 1.

Deposit number- MCEM - BPO 141-143.

Sigalion cirriferum Orensanz and Gianuca, 1974

Sigalion cirriferum Orensanz and Gianuca, 1974: 4, figs. 2-3, 6.

Sigalion cirriferum - Lana, 1984: 36; 1987: 1061, table 1; Amaral and Nallin, 2005: 48, table 2.

Deposit number - MCEM - BPO 140.

Sigalion taquari Amaral and Nonato, 1984

Sigalion taquari Amaral and Nonato, 1984: 23, figs. 44-53.

Sigalion taquari — Lana, 1987: 1061, table 1; 1991: 125, figs. 9-10.

Deposit number - MCEM - BPO 133-139.

Sthenelanella peterseni Lana, 1991

Sthenelanella peterseni - Lana, 1984: 30; 1987: 1061, table 1 (Original name in an upublished thesis).

Sthenelanella peterseni Lana, 1991: 122, figs. 1-8.

Deposit number - MCEM -BPO 130.

Sthenelais limicola (Ehlers, 1864)

Sigalion limicola Ehlers, 1864: 120, figs 4-7.

Sthenelais limicola - Lana, 1984: 42; 1987: 1061, table 1; 1991: 131; Amaral and Nallin, 2005: 48, table 2.

Deposit number - MCEM - BPO 151-169; MZUSP 303.

Sthenelais articulata Kinberg, 1855

Sthenelais articulata Kinberg, 1855: 387.

Sthenelais articulata — Lana, 1984: 43; 1987: 1061, table 1; 1991: 131.

Deposit number - MCEM - BPO 148-150; 231.

Sthenolepis oculata (Hartman, 1942)

Leanira oculata Hartman, 1942 : 93, figs.1-5.

Sthenolepis oculata — Lana, 1984: 45; 1987: 1061, table 1; 1991: 131.

Deposit number - MCEM - BPO 230.

Thalenessa edwardsii (Kinberg, 1855)

Sigalion edwardsii Kinberg, 1855: 387.

Thalenessa edwardsii — Lana, 1984: 38; 1987: 1061, table 1; 1991: 127, figs. 17-21.

Deposit number - MCEM - BPO 144; 232.

\section{Spionidae Grube, 1850}

Aonides mayaguezensis Foster, 1969

Aonides mayaguezensis Foster, 1969: 393, figs. 22-33.

Apoprionospio dayi Foster, 1969

Apoprionospio dayi Foster, 1969: 383, figs. 1-11.

Prionospio dayi - Bolívar, 1986: 40, figs. 89-93; Bolívar and Lana, 1987: 143, figs. 75-79.

Deposit number - USNM 100448

Apoprionospio pygmaea (Hartman, 1961)

Prionospio pygmaeus Hartman, 1961: 93.

Deposit number - MZUSP 59-61.

Boccardiella bihamata Blake and Kudenov, 1978

Boccardiella bihamata Blake and Kudenov, 1978: 265, fig. 48.

Deposit number - MZUSP 68.

Boccardiella ligerica (Ferronnière, 1898)

Boccardia ligerica Ferronnière, 1898: 109, pl. 6, figs. a-i.

Deposit number - MZUSP 70.

Dipolydora barbilla (Blake, 1981)

Polydora barbilla Blake, 1981: 947, fig. 1. 


\section{LISTS OF SPECIES}

Deposit number - MZUSP 80.

Dipolydora socialis (Schmarda, 1861)

Leucodore socialis Schmarda, 1861: 64, figs. a-c, pl. 26, fig. 209, 209a.

Polydora socialis - Bolívar, 1986: 15, figs. 7-13; Bolívar and Lana, 1987: 113, figs. 1-7; Amaral and Nallin, 2005: 48, table 2.

Deposit number - MZUSP 82-90.

Dispio sp.

Deposit number - MZUSP 94-95; 98.

Dispio remanei Friedrich, 1956

Dispio remanei Friedrich, 1956: 65, fig. 6.

Dispio remanei - Bolívar, 1986: 22, figs. 30-42; Bolívar and Lana, 1987: 120, figs. 26-29 Amaral and Nallin, 2005: 48, table 2.

Deposit number - MCEM - BPO 55-57; USNM 100446.

Heterospio sp.

Deposit number - MZUSP 106-107.

Laonice branchiata Nonato, Bolívar and Lana, 1986

Laonice branchiata Nonato, Bolívar and Lana, 1986: 21, figs. 1-7.

Laonice branchiata - Bolívar and Lana, 1987: 120.

Deposit number - MCEM - BPO 52-54; MZUSP 111; 114; 116; USNM 100451.

Laonice sp. 1

Laonice cirrata- Bolívar, 1986: 18, figs. 27-31; Bolívar and Lana, 1987: 118, figs. 21-25. Not Sars, 1851: 64 .

Deposit number - MCEM-BPO 39; 41-44; 48.

Laonice sp. 2

Deposit number - MZUSP 117; 119-120; $122 ; 124-125$.

Microspio pigmentata (Reish, 1959)

Spiophanes pigmentata Reish, 1959: 11, pl. 6, figs. 1-4.

Microspio pigmentata - Bolívar, 1986: 27, figs. 51-55; Bolívar and Lana, 1987: 126, figs. 38-42.

Deposit number - MCEM - BPO 59-60; MZUSP 137.

Paraprionospio sp.

Paraprionospio pinnata - Bolívar, 1986: 35, figs. 73-79; Bolívar and Lana, 1987: 136, figs. 59-65;

Blankensteyn and Moura, 2002: 718, table 2. Not Ehlers, 1901: 163.

Deposit number - MCEM - BPO 78-90; MZUSP 146-151.

Poecilochaetus australis Nonato, 1963

Poecilochaetus australis Nonato, 1963: 17, figs. 1-11.

Poecilochaetus johnsoni - Ribeiro, 2004: 55.

Deposit number- MZUSP 155.

Poecilochaetus sp. n 1 Santos and Mackie (in preparation)

Deposit number- MCEM- BPO 1290-1291.

Poecilochaetus sp. n.2 Santos and Mackie (in preparation)

Deposit number - MCEM-BPO 1292-1294.

Polydora carinhosa Radashevsky, Lana and Nalesso (submitted).

Polydora carinhosa Radashevsky, Lana and Nalesso (submitted).

Polydora colonia Moore, 1907

Polydora colonia Moore, 1907: 199, pl. 15, figs. 18-23.

Deposit number - MZUSP 162-164.

Polydora cornuta Bosc, 1802

Polydora cornuta Bosc, 1802: 150, p. 5, figs 7-8.

Polydora cornuta - Radashevsky, 2005: 3, figs. 1-4.

Deposit number - SMF 13975; 13978; USNM 1020474-1020475. 


\section{LISTS OF SPECIES}

Polydora ecuadoriana Blake, 1983

Polydora ecuadoriana Blake, 1983: 257-258, fig. 26.

Polydora ecuadoriana - Radashevsky, Lana and Nalesso (submitted).

Deposit number - MZUSP 166; 169; 172; USNM 1022185-1022187.

Polydora cf. haswelli Blake and Kudenov, 1978

Polydora haswelli Blake and Kudenov, 1978: 259, fig. 44.

Polydora haswelli - Radashevsky, Lana and Nalesso (submitted).

Polydora websteri - Bolívar, 1986: 17, figs. 14-26; Bolívar and Lana, 1987: 115, figs. 8-20;

Blankensteyn and Moura, 2002: 718, table 2. Not Hartman in Loosanoff and Engle, 1943.

Deposit number - MCEM - BPO 38; MZUSP 174; 176; 178.

Polydora nuchalis Woodwick, 1953

Polydora nuchalis Woodwick, 1953: 381, fig. 1.

Deposit number - MZUSP 180.

Polydora sp. 1

Deposit number - MZUSP 182.

Polydora sp. 2

Deposit number - MZUSP 183-185.

Prionospio ehlersi Fauvel, 1928

Prionospio ehlersi Fauvel, 1928: 10, figs. 1a-e.

Prionospio ehlersi - Bolívar, 1986: 38, figs. 84-88; Bolívar and Lana, 1987: 141, figs. 70-74.

Deposit number - MCEM - BPO 92.

Prionospio heterobranchia Moore, 1907

Prionospio heterobranchia - Moore, 1907: 195, pl. XV, figs. 1-6.

Deposit number - MZUSP 200.

Prionospio sp.

Prionospio steenstrupi - Bolívar, 1986: 37, figs. 80-83; Bolívar and Lana, 1987: 139, figs. 66-69. Not

Deposit number - MCEM - BPO 91.

Pseudopolydora achaeta Radashevsky and Hsieh, 2000

Pseudopolydora achaeta Radashevsky and Hsieh, 2000: 223, figs. 4, 5, 11a.

Deposit number - MZUSP 237; 240.

Pseudopolydora diopatra Hsieh, 1992

Pseudopolydora diopatra Hsieh, 1992: 630, figs. 1-10.

Deposit number - IMBV 12394.

Scolelepis goodbodyi (Jones, 1962)

Nerinides goodbodyi Jones, 1962: 187, figs. 66-68.

Scolelepis squamata - Bolívar, 1986: 25, figs. 43-50; Bolívar and Lana, 1987: 124, figs. 30-37;

Blankensteyn and Moura, 2002: 718, table 2. Not Lumbricus squamatus Müller, 1806; 39.

Deposit number - MCEM - BPO 58; MZUSP 237; 240.

Scolelepis sp. 1

Deposit number - MZUSP 259-260.

Scolelepis sp. 2

Deposit number - MZUSP 249.

Scolelepis quinquedentata (Hartmann-Schröder, 1965)

Nerinides quinquedentata Hartmann-Schröder, 1965: 203, figs. 187-190.

Deposit number - MZUSP 255; 257.

Spiophanes anoculata Hartman, 1960

Spiophanes anoculata Hartman, 1960: 118.

Spiophanes bombyx - Bolívar, 1986: 33, figs. 67-72; Bolívar and Lana, 1987: 134, figs. 54-58. Not

Claparède, 1870.

Deposit number - MCEM - BPO 74-77; MZUSP 268-270; 272-277. 


\section{LISTS OF SPECIES}

Spiophanes berkeleyorum Pettibone, 1962

Spiophanes berkeleyorum Pettibone, 1962: 78, figs. 1-4.

Spiophanes cf. kroyeri - Bolívar, 1986: 30, figs. 56-61; Bolívar and Lana, 1987: 129, figs. 43-47. Not Grube, 1860.

Deposit number - MCEM - BPO 61; 62; MZUSP 278-294.

Spiophanes duplex (Chamberlin, 1919)

Morants duplex Chamberlin, 1919: 17.

Spiophanes missionensis - Bolívar, 1986: 32, figs. 62-66; Bolívar and Lana, 1987: 132, figs. 48-53;

Blankensteyn and Moura, 2002: 718, table 2. Not Hartman, 1941.

Deposit number - MCEM - BPO 63-73; ZSRO P1413; P1576-P1577; USNM 100450.

Streblospio sp.

Deposit number - MZUSP 304; 306-307.

\section{Sternaspidae Carus, 1863}

Sternaspis capillata Nonato, 1966

Sternaspis capillata Nonato, 1966: 79-84, figs. 1-9.

Sternaspis capillata - Nonato, 1981: 188; Attolini, 2002: 28, figs. 8-9, 11-12, tables 4, 8.

\section{Syllidae Grube, 1850}

Exogone arenosa Perkins, 1980

Exogone arenosa Perkins, 1980: 1094, figs. 5g-j, 6.

Exogone arenosa - Lana, 1984: 66, figs. 55-56; 1987: 1061, table 1.

Langerhansia cornuta (Rathke, 1843)

Syllis cornuta Rathke, 1843: 164.

Langerhansia cornuta — Lana, 1987: 1061, table 1.

Odontosyllis heterofalchaeta Temperini, 1981

Odontosyllis heterofalchaeta Temperini, 1981: 22, figs. 44-48 176 (Original name in an unpublished thesis)

Odontosyllis heterofalchaeta - Lana, 1984: 69, figs. 59-60.

Odontosyllis fulgurans (Audouin and Milne-Edwards, 1834)

Syllis fulgurans Audouin and Milne-Edwards, 1834: 229.

Odontosyllis fulgurans - Amaral et al., 2006: 147.

Pionosyllis pectinata Temperini, 1981

Pionosyllis pectinata Temperini, 1981: 23, figs. 49-55. (Original name in an unpublished thesis).

Pionosyllis pectinata — Lana, 1984: 67, figs. 57-58; 1987: 1061, table 1.

Deposit number - MCEM - BPO 1449-1450; 1452-1454; 1461.

Typosyllis hialina (Grube, 1863)

Syllis hialina Grube, 1863: 45.

Typosyllis hialina - Lana, 1984: 71, fig. 61; 1987: 1061, table 1.

Deposit number-MCEM -BPO 1446.

Obs: This is probably a misidentification and this material needs to be revised.

Typosyllis variegata (Grube, 1860)

Syllis variegata Grube, 1860: 65.

Typosyllis variegata — Lana, 1984: 72; 1987: 1061, table 1.

\section{Terebellidae Grube, 1850}

Artacama benedeni Kinberg, 1867

Artacama benedeni Kinberg, 1867: 346. 


\section{LISTS OF SPECIES}

Artacama benedeni - Blankensteyn, 1988: 32, figs. 5a-f, 9.

Deposit number - MCEM - BPO 252-259.

Loimia medusa (Savigny, 1818)

Terebella medusa Savigny, 1818: 95.

Loimia medusa - Blankensteyn, 1988: 58, figs. 12a-f, 17.

Deposit number - MCEM - BPO 288-299; MZUSP 127.

Obs: This is probably a misidentification and this material needs to be revised.

Loimia grubei (Grube, 1878)

Terebella montagui Grube, 1878: 1.

Loimia grubei - Blankensteyn, 1988: 62, figs. 13a-h, 16.

Deposit number - MCEM - BPO 301-304.

Obs: This is probably a misidentification and this material needs to be revised.

Lysilla pacifica Hessle, 1917

Lysilla pacifica Hessle, 1917: 232, fig. 66.

Lysilla pacifica - Blankensteyn, 1988: 91, figs. 22a-c, 24.

Deposit number - MCEM - BPO 322-323.

Obs: This is probably a misidentification and this material needs to be revised.

Neoamphitrite variabilis (Risso, 1826)

Terebella variabilis Risso, 1826: 408

Neoamphitrite cf. variabilis - Blankensteyn and Moreno, 1988: 32, figs. 6a-g, 9.

Obs: This is probably a misidentification and this material needs to be revised.

Nicolea uspiana (Nogueira, 2003)

Paraeupolymnia uspiana Nogueira, 2003: 405, figs. 1-2

Nicolea venustula - Blankensteyn \& Moreno, 1999: 320, fig. 1.

Deposit number - MZUSP 142.

Octobranchus longipes Blankesteyn and Lana, 1987

Octobranchus longipes Blankesteyn and Lana, 1987: 671, figs. 1a-c., 2a-b, 3a-e.

Octobranchus longipes — Blankensteyn, 1988: 104, figs. 26a-g, 29.

Deposit number - MCEM - BPO 247-248.

Pista corrientis McIntosh, 1885

Pista corrientis McIntosh, 1885: 457, fig. 11, pl. 48, fig. 35, pl. 27 a.

Pista corrientis - Blankensteyn, 1988: 43, figs. 10a-g, 16, 17.

Deposit number - MCEM - BPO 269-286.

Pista cristata (Müller, 1776)

Amphitrite cristata Müller, 1776: 216.

Pista cristata - Blankensteyn, 1988: 47, figs. 8a-g, 9.

Deposit number - MCEM - BPO 264-268.

Obs: This is probably a misidentification and this material needs to be revised.

Terebellides anguicomus Müller, 1858

Terebellides anguicomus Müller, 1858: 218, pl. 7, figs. 22-25.

Terebellides anguicomus - Blankensteyn, 1988: 107, figs. 27a-g, 29, 30; Solis-Weiss et al., 1991: 149,

fig. 1g-I; Garraffoni \& Lana, 2003: 360, figs. 11,-12.

Deposit number - MCEM - BPO 249; 250; 328-334; MZUSP 308.

Thelepus setosus (Quatrefages, 1865)

Phenacia setosus Quatrefages, 1865: 374.

Thelepus setosus - Blankensteyn, 1988: 74, figs. 17, 18a-g, 24.

Deposit number - MCEM - BPO 309-313.

Obs: This is probably a misidentification and this material needs to be revised. 


\section{LISTS OF SPECIES}

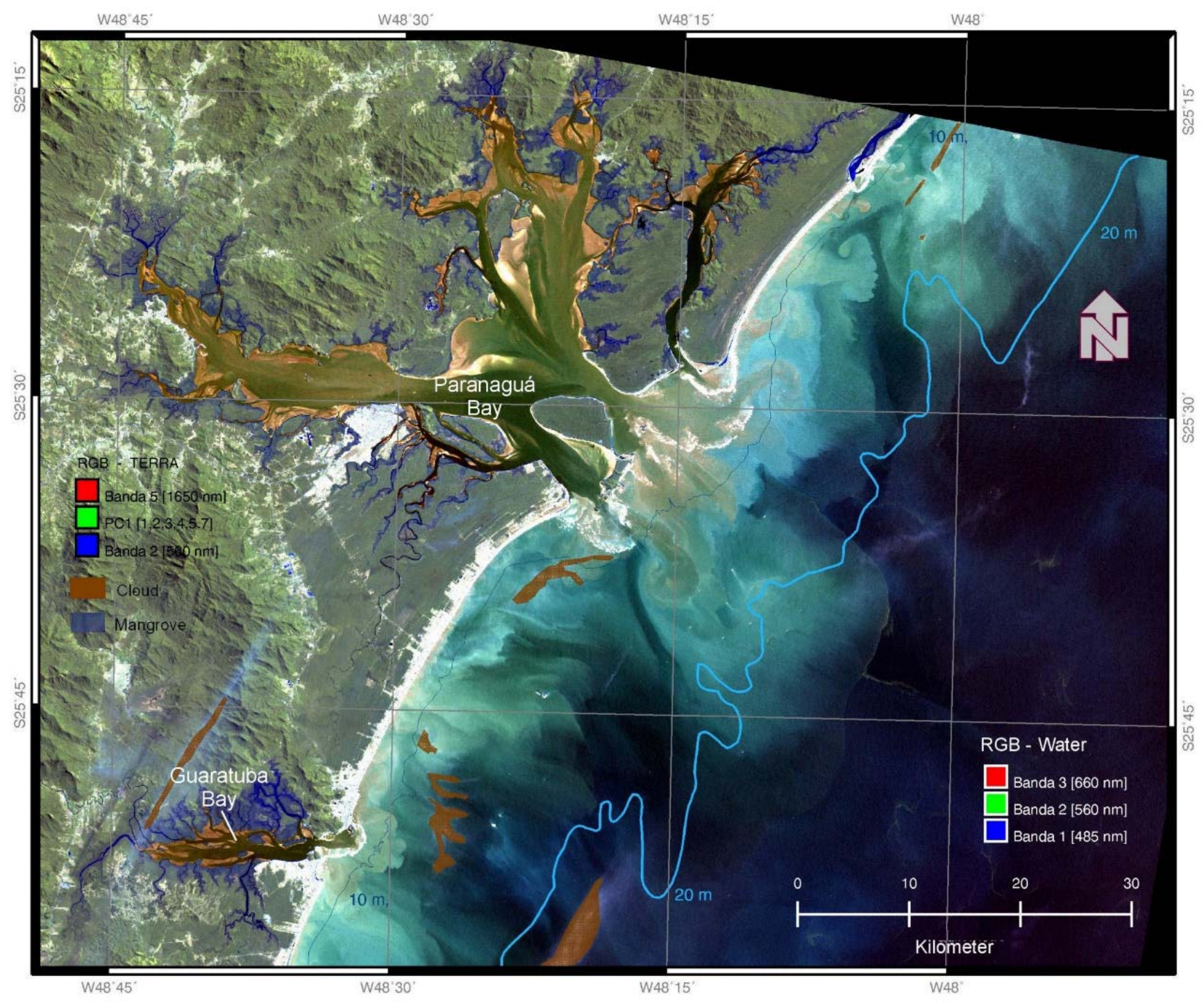

Figure 1. Location of sampled environments in Paraná State.

\section{Acknowledgements}

We would like to thank Maurício Noernberg for kindly providing the map with sampling areas (Figure 1), and Alexandra Rizzo and an anonymous reviewer for advice and comments.

\section{Literature cited}

Aiyar R. G. and K. H. Alikunhi. 1940. On a new Pisionid from the sandy beach, Madras. Records of Indian Museum 42: 89-107.

Amaral A. C. Z. and E. F. Nonato. 1982. Anelídeos poliquetos da costa brasileira. Aphroditidae e Polynoidae. Conselho Nacional de Desenvolvimento Científico e Tecnológico 3: 7-46.
Amaral A. C. Z. and E. F. Nonato. 1984. Anelídeos Poliquetos da costa brasileira. 4 Polyodontidae, Pholoidae, Sigalionidae e Eulepethidae. Conselho Nacional de Desenvolvimento Científico e Tecnológico 4: 01-54.

Amaral, A. C. Z., P. C. Lana, A. E. Rizzo, T. M. Steiner, E. V. Pardo, C. S. G. Santos, A. C. Carvalho, M. Wagner, R. A. Fernanda, A. R. S. Garraffoni, A. C. Carvalho, A. C. Brasil, Z. Ribeiro, J. M. M. Nogueira, A. Abbud, M. Rossi and M. Fukuda. 2004. Filo Polychaeta. Pp. 114-125, In: A. C. Z. Amaral and C. L. B. Rossi-Wongtschowski, 


\section{LISTS OF SPECIES}

(Eds.). Biodiversidade bentônica da região sudeste-sul do Brasil - plataforma externa e talude superior. Série Documentos REVIZEE - Score Sul.

Amaral, A. C. Z. and S. Nallin. 2005. Catálogo das espécies dos Anellida Polychaeta da costabrasileira. EletronicDatabase accessible at:http://www.ib.unicamp.br/destaques/biota/ bentos_marinho/7.htm. Captured on 18 april 2005.

Amaral, A. C. Z., A. E. Rizzo and E. P. Arruda. 2006. Manual de identificação dos invertebrados marinhos da região sudestesul do Brasil. São Paulo, EDUSP.

Augener, H. 1934. Polychaeten aus den Zoologischen Museen von Leidenund Amsterdam. Zoologische Mededelengen 17: 67-160.

Attolini, F. 2002. Padrões de distribuição de poliquetas macrobentônicos na região de plataforma externa e talude superior entre Cabo Frio (RJ) e Cabo de Santa Marta (SC), costa sudeste do Brasil. Unpublished thesis, Instituto Oceanográfico, Universidade de São Paulo, Brazil.

Audouin, J. V. and H. M. Milne-Edwards. 1833. Classification des Annelides, et description de celles qui habitent les cotes de la France. Annales des sciences naturelles 28: 187-247.

Audouin, J. V. and H. M. Milne-Edwards. 1834. Recherches pour servir a l'histoire naturelle du littoral de la France, ou Recueil de memoires sur l'anatomie, la physiologie, la classification et les moeurs des animaux de nos cotes; ouvrage accompagne de planches faites d'apres nature. Paris, Crochard.

Berkeley, E. and C. Berkeley. 1939. On a collection of Polychaeta, chiefly from the west coast of Mexico. Annals and Magazine of Natural History 3: 321-346.

Bhaud, M. 1998. Developmetnal pattern diversity on a latitudinal transect: interation of adut habitat and larval dispersal. Océanis 24: 117-136.

Bhaud, M. and A. V. Petti-Monica. 2001. Spiochaetopterus nonatoi, a new species of Chaetopteridae (Polychaeta) from Brazil: biogeographical consequences. Journal of Marine Biological Assossiaiton of United Kingdom 81: 225-234.
Blainville, H. D. 1828. Dictionnaire des Sciences naturelles, dans lequel on traite methodiquement des differens etres de la nature, consideres soit en eux-memes, d'apres l'etat actuel de nos connaisance, soit relativement a l'utilite qu'en peuvent retirer la medicine, l'agricu. Stratsbourg.

Blake, J. A. 1981. Polydora and Boccardia species (Polychaete: Spionidae) from Western Mexico, chiefly from calcareous habitats. Proceedings of biological Society of Washington 93: 947-962.

Blake, J. A. 1983. Polychaetes of the family Spionidae from South America, Antarctica and adjacent seas and islands. Biology of the Antarctic Seas XIV Antarctic Research Series 39: 205-288.

Blake, J. A. and J. D. Kudenov. 1978. The Spionidae (Polychaeta) from southeastern Australia and adjacent areas with a revision of the genera. Memoirs of the National Museum of Victoria 39: 171-280.

Blanchard, E. 1849. Anelidos do Chile. In: Gay's historia fisica politica de Chile. Segun adquiridos en esta republia durante doce años de residencia en ella. Zoologia 3: 9-52.

Blankensteyn, A. 1988. Terebellidae e Trichobranchidae (Annelida: Polychaeta) da costa sudeste do Brasil $\left(24^{\circ}-27^{\circ} \mathrm{S}\right)$. Unpublished thesis. Departamento de Zoologia, Universidade Federal do Paraná, Curitiba, Brazil.

Blankensteyn, A. and P. C. Lana. 1987. Octobranchus longipes $\mathrm{sp} . \mathrm{n}$. (Trichobranchidae: Polychaeta) da costa sudeste do Brasil. Arquivos de Biologia e Tecnologia 30: 671-676.

Blankensteyn, A. and R. S. Moura. 2002. Lista preliminar das espécies da macrofauna de fundos inconsolidados da Baía de Guaratuba, Paraná, Brasil. Revista Brasileira de Zoologia 19: 715-721.

Blankensteyn, A. and T. R. Moreno. 1999. Nova ocorrência de Nicolea venustula (Montagu) (Polychaeta, Terebellidae) na costa sul do Brasil. Revista Brasileira de Zoologia 16 (1): 319-322.

Böggemann, M. 2002. Revision of the Glyceridae Grube, 1850 (Annelida, Polychaeta). 


\section{LISTS OF SPECIES}

Abhandlungen der Senckenbergergischen Naturforschenden Gesellschaft 555: 1-249.

Böggemann, M. 2005. Revision of the Goniadidae (Annelida, Polychaeta). Abhandlungen des Naturwissenschaftlichen Vereins in Hamburg 1-354.

Bolívar G. A. 1986. Padrões de distribuição de Spionidae e Magelonidae (Annelida: Polychaeta) do litoral do Estado do Paraná. Unpublished thesis. Departamento de Zoologia, Universidade Federal do Paraná, Curitiba, Brazil.

Bolívar G. A. 1990. Orbiniidae, Paraonidae, Heterospionidae, Cirratulidae, Capitellidae, Maldanidae, Scalibregmidae e Flabelligeridae (Annelida: Polychaeta) da costa sudeste do Brasil (22 $2^{\circ} 57^{\prime} \mathrm{S}-27^{\circ} 20^{\prime}$ S). Unpublished thesis. Departamento de Zoologia, Universidade Federal do Paraná, Curitiba, Brazil.

Bolívar G. A. and P. C. Lana. 1986. Magelonidae (Annelida, Polychaeta) do litoral sudeste do Brasil. Nerítica 1(3):131-147.

Bolívar G. A. and P. C. Lana. 1987. Spionidae (Annelida, Polychaeta) do litoral do estado do Paraná. Nerítica 1(3):107-148.

Bosc L. A. 1802. Histoire naturelles des vers, contenant leur description et leur moeurs avec figures dessinees d'apres nature. Paris, Deterville.

Camargo, M. G. 1993. Lumbrineridae (Annelida: Polychaeta) da costa Sul e Sudeste do Brasil. Unpublished thesis. Departamento de Zoologia, Universidade Federal do Paraná, Curitiba, Brazil.

Camargo, M. G. and P. C. Lana. 1995a. Lumbrineridae (Polychaeta: Eunicemorpha) da costa Sul e sudeste do Brasil, I. Lysarete, Arabellonereis, Lumbrineriopsis, Lumbrinerides, Paraninoe e Ninoe. Iheringia 79: $77-$ 91.

Camargo, M. G. and P. C. Lana. 1995b. Lumbrineridae (Polychaeta: Eunicemorpha) da costa Sul e sudeste do Brasil, II. Lumbrineris. Iheringia 79: 93-120.

Camargo, M. L. Z. 1955. Sobre a ocorrência de Chaetopterus no Paraná. Dusenia 1/2: 61-67.

Carrera-Parra, L. F. 2004. Revision of Lumbricalus (Polychaeta: Lumbrineridae). Journal of the Marine Biological Association of the United Kingdom 84:81-91.
Chamberlin, R. 1919. The Annelida Polychaeta. Memomirs of comparative Zoology of Harvard 48: 1-514.

Claparede, E. 1868. Les annelides chetopodes du Golfe de Naples. Premiere Partie. Mémoires de la Société de physique et d'histoire naturelle de Genève 19: 313-584.

Claparede, E. 1870. Les annelides chetopodes du Golfe de Naples. Supplement. Mémoires de la Société de physique et d'histoire naturelle de Genève 20: 365-542.

Day, J. H. 1961. The Polychaete Fauna of South Africa. Part 6. Sedentary species dredged off Cape coasts with a few new records from the shore. Journal of the Linnean Society of London 44: 463-560.

Day, J. H. 1963. Polychaete Fauna of South Africa: Part 7. Species from depths between 1,000 and 3,330 meters west of Cape Town. Annals of the South African Museum 46: 353-371.

Day, J. H. 1967. A monograph on the Polychaeta of Southern Africa. British Museum of Natural History: 656: 1-878.

Day, J. H. 1973. New polychaeta from Beaufort, with a key to all species recorded from North Carolina. NOAA Technical Reports NMFS CIRC-375: 1-140.

Delle Chiaje, S. 1828. Memorie sulla storia e notomia degli animali senza vertebre del regno de Napoli. Naples 3: 1-232.

Delle Chiaje, S. 1829. Memorie sulla storia e notomia degli animali senza vertebre del regno de Napoli. Naples 4: 1-214.

Delle Chiaje, S. 1844. Descrizione e notomia degli animali invertebrati della Sicília citeriore osservati vivi negli anni 1822-30. Napoli.

Ehlers, E. 1868. Die Borstenwurmer (Annelida Chaetopoda) nach systematischen und anatomischen Untersuchungen dargestellt. Leipzig, Wilhelm Engelmann.

Ehlers, E. 1887. Report on the annelids of the dredging expedition of the U. S. coast survey steamer Blake. Memoris of comparative Zoology of Harvard 15: 1-335.

Ehlers, E. 1897. Polychaeten. Hamburger Magellanische Sammelreise 1897:1-148.

Ehlers, E. 1901. Die polychaeten des Magellanischen Anneliden und chilenischen strandes. Nachrichte de Koniglichen 


\section{LISTS OF SPECIES}

Gesellschaft der Wissenschaften Goettingen Mathematisch-Physikalische Klasse 232 p.

Ehlers, E. 1908. Die bodensassigen anneliden aus des Sammlungen der deutschen tiefseeExpedition. Wissenschaftliche Ergebnisse der deustschen tiefsee-Expedition auf dem Dampfer Valdivia 1898-1899 16: 1-168.

Eisig, H. 1914. Zur Systematik, Anatomie und Morphologie der Ariciiden nebst Beitragen zur generellen Systematik. Mitteilungen aus der Zoologischen Station zu Neapel 21: 153-600.

Elias, R. and C. S. Bremec. 2003. First record of the genus Armandia (Opheliidae, Polychaeta) in Argentine waters, with the description of Armandia loboi sp n. Bulletin of Marine Science 72: 181-186.

Elias, R., C. S. Bremec, P. C. Lana and J. M. Orensanz. 2003. Opheliidae (Polychaeta) from the Southwestern Atlantic Ocean, with the description of Travisia amadoi n. sp., Ophelina gaucha $\mathrm{n}$. sp. and Ophelina alata n. sp. Hydrobiologia 496: 75-85.

Fabricius, O. 1780. Fauna Groenlandica, systematice sistens, Animalia Groenlandiae occidentalis hactenus indagata, quoad nomen specificum, triviale, vernaculumque synonyma auctorum plurium, descriptionem, locum, victum, generationem, mores, usum, capturamque singuli prout de. Hafnie et Lipsia.

Fauchald, K. 1982. Revision of Onuphis, Nothria, and Paradiopatra (Polychaeta: Onuphidae) based upon type material. Smithsonian Contributions to Zoology 356: 1-109.

Fauvel, P. 1913. Campagne du Pourquoi-Pas? (Islande et Jan Mayen, 1912). Annelides Polychetes. Bulletin du Muséum d'Histoire Naturelle 2: 80-93.

Fauvel, P. 1928. Annelides Polychetes nouvelles du Maroc. Bulletin de la Société Zoologique de France 53: 1-9.

Ferronnière, G. 1898. Contribution à l'étude de la faune de la Loire-inferieure (Polygordiens, Spionidiens, Nemertien). Bulletin de la Société des Sciences Naturelles de l'Ouest de la France 8: 101-115.

Foster, N. M. 1969. New species of spionids (Polychaeta) from the Gulf of Mexico and Caribbean Sea with a partial revision of the genus Prionospio. Proceedings of biological Society of Washington 82: 381-400.

Frey, H. and R. Leuckart. 1847. Beiträge zur Kenntnis Wirbelloser Thiere mit Besonderer Berücksichtigung der Fauna des Norddeutschen Meeres. Braunschweig, Friedrich Vieweg und Sohn.

Friedrich, H. 1956. Mitteilungen uber neue und wenig bekannte Polychaeten aus Mittel- und Sudamerika. Senckenberg Biologue 37(1-2): 57-68.

Garraffoni, A. R. S. and P. C. Lana. 2003. Species of Terebellides (Polychaeta, Terebellidae, Trichobranchinae) from the Brazilian coast. Iheringia 93: 355-363.

Glasby, C. J. 1999. The Namanereidinae Polychaeta, Nereididae). Part 1 Taxonomy and Phylogeny. Part 2 Biogeography. Records of the Australian Museum 25: 1129.

Grube A. E. 1840. Actinien, Echinodermen und Würmer des Adriatischen- und Mittelmeers nach eigenen Sammlungen beschreiben Königsberg, J. H. Bon.

Grube, A. E. 1856. Annulata Orstediana. Enumeratio Annulatorum, quae in itinere per Indiam occidentalem et Americam centralem annis 1845-1848 suscepto legit cl. A. S. Orsted, adjectis speciebus nonnullis a cl. H. Kroyero in itinere ad Americam meridionalem collectis. Videnskabelige Meddelelser fra Dansk naturhistorisk Forening i Köbenhavn 1856: 44-62.

Grube, A. E. 1857. Annulata Orstediana. Enumeratio Annulatorum, quac in itinere per Indiam occidentalem et Americam centralem annis 1845-1848 suscepto legit cl. A. S. Orsted, adjectis speciebus nonnullis a cl. H. Kroyero in itinere ad Americam meridionalem collectis. Videnskabelige Meddelelser fra Dansk naturhistorisk Forening i Köbenhavn 1857: 158-166.

Grube, A. E. 1870. Beschreibung neuer oder weniger bekannter von Hrn. Prof. Ehrenberg gesammelter Anneliden des rothen Meeres. Monatsbericht der Koniglich Preusischen Akademie der Wissenschaften zu Berlin: 484-521.

Grube, A. E. 1870. Über die Goldkronchen (Sabellarien oder Hermellen). Schlesischen 


\section{LISTS OF SPECIES}

gesellschaft für vaterlandische Kultur Breslau Jahresber. 47: 69-70.

Grube, A. E. 1871. Nachtraege zur vorigem und Vorlage einer Lumbriconereis gigantea einer neuen Oenone und Serolis. Schlesischen gesellschaft fur vaterlandische Kultur Breslau Jahresber. 49:56-58.

Grube, A. E. 1872. Über die Gattung Lycastis und ein paar neue Arten derselben. Schlesischen gesellschaft für vaterlandische Kultur Breslau Jahresber. 49: 47-48.

Grube, A. E. 1874. Descriptiones Annulatorum novorum mare Ceylonicum habitantium ab honoratissimo Holdsworth collectorum. Prooceedings of the Zoological Society of London 1874: 325-329.

Grube A. E. 1878. Untersuchungen über die Familie Eunicea. Jahresbericht der Schlesischen Gesellschaft für vaterländische Kultur 55: 79-104.

Gruet, Y. and P. C. Lana. 1988. Remarks on the opercular paleae Sabellaria bella Grube, 1870 and Sabellaria bellis Hansen, 1882 (Sabellariidae; Polychaeta) from the Southeast coast of Brazil. Nerítica 3: 31-36.

Hansen, G. A. 1878. Annelider fra den norske Nordhavsexpedition i 1876. Nyt Magazin for Naturvidenskaberne 24:1-17.

Hansen, A. 1882. Recherches sur les Annélides recueilles par M. le professeur Édouard Van Beneden pendant voyage au Brésil et a la Plata. Memoires de l'Institut Royal des Sciences Naturelles de Belgiques 44: 1-29.

Hartman, O. 1938. Nomenclatural changes involving California polychaete worms. Journal of the Washington Academy of Sciences 26: 31-32.

Hartman, O. 1941. Some contributions to the biology and life history of Spionidae from California. With keys to species and genera and descriptions of two new forms. Allan Hancock Pacific Expedition 7: 289-323.

Hartman, O. 1942. The identity of some marine annelid worms in the United States National Museum. Proceedings of United States Natural Museum 92: 101-140.

Hartman, O. 1944. Polychaetous Annelids. Part V. Eunicea. Allan Hancock Pacific Expedition 10: 1-237.
Hartman, O. 1947. Polychaetous annelids. Part VII. Capitellidae. Allan Hancock Pacific Expedition 10: 391-481.

Hartman, O. 1950. Polychaetous annelids. Goniadidae, Glyceridae and Nephtyidae. Allan Hancock Pacific Expedition 15: 1-181.

Hartman, O. 1951. The littoral marine annelids of the Gulf of Mexico. Publications of the Institute of Marine Science 2: 7-124.

Hartman, O. 1953. Non-pelagic polychaeta of the Swedish Antarctic Expedition 1901-1903. Further Zoological Results of the Swedish Antarctic Expedition 1901-1903 4: 1-83.

Hartman, O. 1959. Capitellidae and Nereidae (marine Annelids) from the gulf side of Florida with a review of freshwater Nereidae. Bulletin of the Marine Science of the Gulf Caribbean 9: 153-168.

Hartman, O. 1960. Systematic account of some marine invertebrate animals from the deep basins of Southern California. Allan Hancock Pacific Expedition 22: 69-216.

Hartman, O. 1961. Polychaetous annelids from California. Allan Hancock Pacific Expedition 25: 1-226.

Hartman,O.1965.Deep-waterbenthic polychaetous annelids off New England to Bermuda and other North Atlantic areas. Occasonal Paper of Allan Hancock Foundation 28: 1-378.

Hartman, O. 1943. Description of Polydora websteri. In: Loosanoff, V.L. and J.B. Engle, Polydora in oysters suspended in the water. Bulletin of Marine Biology 85: 69-78.

Hartmann-Schröder, G. 1956. Neue Armandiaarten (Opheliidae, Polychaeta) aus Brasilien und El Salvador. Beitrage zur neotro Fauna 1: 63-68.

Hartmann-Schröder, G. 1965. Die Polychaeten des Sublitorals Zur Kenntnis des Sublitorals der Chilenischen Küste unter besonderer Berücksichtigung der Polychaeten und Ostracoden. T1.II. Die Polychaeten des Sublitorals. Mitteilungen aus dem Hamburgischen zoologischen Museum und Institut 62: 59-305.

Hartman, O. and K. Fauchald. 1971. Deep-water benthic polychaetous annelids off New England to Bermuda and other North Atlantic areas. Part II. Allan Hancock Monography of marine Biology 6: 1-327. 


\section{LISTS OF SPECIES}

Hessle, C. 1917. Zur Kenntnis der terebellomorphen Polychaeten. Zoologiska bidrag från Uppsala 5: 39-258.

Hoagland, R. A. 1919. Polychaetous Annelids from Porto Rico, the Florida Keys and Bermuda. Bulletion of American Museum of natutal History 41: 517-591.

Hsieh, H. L. 1992. Pseudopolydora diopatra, a new species (Polychaeta: Spionidae) from Taiwan. Proceedings of the biological society of Washington 105: 630-635.

Jones, M. L. 1962. On some polychaetous annelids from Jamaica, the West Indies. Bulletin of American Museum of Natutal History 124: 169-212.

Jones, M. L. 1963. Four new species of Magelona (Annelida, Polychaeta) and a redescription of Magelona longicornis Johnson. American Museum Novitties 2164: 1-31.

Jones, M. L. 1968. Paraonis pygoenigmatica new species, a new annelid from Massachusetts (Polychaeta: Paraonidae). Proceedings of the biological society of Washington 81 : 323-334.

Jumars, P. A. 1974. A generic revision of the Dorvilleidae (Polychaeta) with six new species from the deep North Pacific. Zoological Journal of the Linnaean Society 54: 101-135.

Kinberg, J. G. H. 1855. Nya slagter och arter af Annelider. Öfversigt af Königlich Vetenskapsakademiens förhandlingar 12: 381-388.

Kinberg, J. G. H. 1858. Part 3: Annulater. Konglia Svenska Fregaten eugenies Resa Omring Jorden under Befal af C. A. Virgin Aren 1851-1853. Vetenskapliga Iakttagelser Zoology 2: 1-32.

Kinberg, J. G. H. 1865. Annulata nova. Öfversigt af Königlich Vetenskapsakademiens förhandlingar 21: 559-574.

Kinberg, J. G. H. 1866. Annulata Nova. Öfversigt af Königlich Vetenskapsakademiens förhandlingar 22: 239-258.

Kinberg, J. G. H. 1866. Annulata nova. Öfversigt af Königlich Vetenskapsakademiens förhandlingar 22: 167-179.

Kinberg, J. G. H. 1867. Annulata nova Öfversigt af Königlich Vetenskapsakademiens förhandlingar 23: 337-357.
Kirkegaard, J. B. 1959. The Polychaeta of West Africa Part I. Sedentary species. Atlantide Report 5: 7-117.

Kirtley, D. W. 1994. A review and taxonomic revision of the family Sabellariidae Johnston, 1865 (Annelida, Polychaeta). Florida, Sabecon Press.

Lana, P. C. 1984. Anelídeos poliquetas errantes do litoral do Estado do Paraná. Unpublished thesis, Instituto Oceanográfico, Universidade de São Paulo, São Paulo, Brazil.

Lana, P. C. 1986. Nephtyidae (Annelida; Polychaeta) do litoral do estado do Paraná (Brasil). Nerítica 1(1): 135-154.

Lana, P. C. 1987. Padrões de distribuição geográfica dos poliquetas errantes (Annelida: Polychaeta) do estado do Paraná. Ciência e Cultura 39: 1060-1063.

Lana, P. C. 1991. Onuphidae (Annelida: Polychaeta) from Southeastern Brazil. Bulletin of Marine Science 48: 280-295.

Lana, P. C. 1991. Sigalionidae (Polychaeta) from the coast of Parana (SE Brazil) and adjacent areas. Ophelia 5: 121-132.

Lana, P. C. and C. S Bremec. 1994. Sabellariidae (Annelida: Polychaeta) from South America. Mémoires du Muséum National D'histoire Naturelle 162: 209-222.

Lana, P. C. and H. H. Sovierzoski. 1987. Neanthes bruaca sp. n. e Nereis broa sp. n. (Nereididae: Polychaeta) da costa sudeste do Brasil. Arquivos de Biologia e Tecnologia 30: 677-688.

Lana, P. C. and Y. Gruet. 1989. Sabellaria wilsoni sp. n. (Polychaeta, Sabellariidae) from the Southeast coast of Brazil. Zoologica Scripta 18: 239-244.

Linnaeus, C. 1761. Fauna Svecica Sistens Animalia Sveciae Regni: Mammalia, aves, amphibia, pisces, insecta, vermes. Distributa per classes and ordines, genera and species, cum differentiis specierum, synonymis auctorum, nominibus incalorum, locis natalium, descriptionibus insectorum. Stockholm, Editio altera.

Leidy, J. 1855. Contributions towards acknowledge of the marine invertebrate fauna of the coast of Rhode Island and New Jersey. Journal of Academy of Natural Science of Philadelphia 3: 135-158. 


\section{LISTS OF SPECIES}

Loosanoff, V. L. and J. B. Engle. 1943. Polydora in oysters suspended in the water. Biological Bullettion of marine Biology 85 : 69-78.

Lorenzi, L. 1998. Composição e distribuição da macrofauna bêntica em gamboas da Baía de Paranaguá (Paraná, Brasil). Unpublished thesis. Departamento de Zoologia. Universidade Federal do Paraná, Curitiba, Brazil.

Lorenzi, L. 2004. Estrutura das associações infaunais sublitorais de substrato inconsolidado adjacente a recifes artificiais e naturais (Paraná, Brasil). Unpublished thesis. Departamento de Zoologia. Universidade Federal do Paraná, Curitiba, Brazil.

Malmgren, A. J. 1865. Nordiska Hafs-Annulater. Öfversigt af Königlich Vetenskapsakademiens förhandlingar 21/22: 51-110,181-192, 355-1410.

Malmgren, A. J. 1867. Annulata Polychaeta Spetsbergiae, Groenlandiae, Islandiae et Scandinaviae hactenus cognita. Öfversigt af Königlich Vetenskapsakademiens förhandlingar 24: 127-235.

Mangum, C. P. 1966. Two new species of Clymenella (Polychaeta: Maldanidae) from Brazil. Postilla 104: 10.

Marenzeller, E. V. 1879. Sudjapanische Anneliden. I. Amphinomea, Aphroditea, Lycoridea, Phyllodocea, Hesionea, Syllidea, Eunicea, Glycerea, Sternaspidea, Chaetopterea, Cirratulea, Amphictenea. Denkschriften der Mathematisch und naturwissenschaftlichen classe der Kaiserliche Akademie der Wissenschaften 41: 109-154.

McIntosh, W. C. 1869. On the structure of British nemerteans, and some new British Annelids. Transactions of the Royal Society of Edinburgh 25: 305-333.

McIntosh, W. C. 1879. On the Annelida obtained during the cruise of H.M.S. 'Valorous' to Davis Strait in 1875. Transactions of the Linnean Society of London 1: 499-511.

McIntosh, W. C. 1885. Report on the Annelida Polychaeta collected by H.M.S. Challenger during the years 1873-1876. Report on the Scientific Results of the Voyage of H.M.S. Challenger during the years 1872-76, Zoology 12: 1-554.
McIntosh, W. C. 1910. A monograph of British Annelids. Vol. Polychaeta. Syllidae to Ariciidae. Royal Society of London 235524.

Nogueira, J. M. M. 2003. A new species of Paraeupolymnia Young and Kritzler, 1986 (Polychaeta: Terebellidae: Terebellinae). Scientia Marina 67: 403-411

Monro, C. C. 1933. The polychaeta sedentaria collected by Dr. C. Crossland at Colon, in the Panama region, and the Galapagos Islands during the expedition of the S.Y.'St. George'. Proceedings of zoological Society of London 1933: 1039-1092.

Monro, C. C. 1937 . On some freshwater Polychaetes from Uruguay. Annals and Magazine of Natural History 20: 241-250.

Monro, C. C. 1937. Polychaeta. The John Murray Expedition Scientific Reports 4: 243-321.

Montagu, G. 1804. Descriptions of several marine animals found on the South coast of Devonshire. Transactions of the Linnean Society of London 7: 61-85.

Montagu, G. 1815. Descriptions of several new or rare animals principally marine, found on the south coast of Devonshire. Transactions of the Linnean Society of London 11:18-21.

Montagu, G. 1818. Descriptions of five British species of the genus Terebella. Transactions of the Linnean Society of London 12: 340344.

Moore, J. P. 1908. Some polychaetous annelids of the northern Pacific coasts of North America. Proceedings of the academy of Natural History of Philadelphia 60: 321364.

Moore, J. P. 1911. The polychaetous annelids dredged by the U.S.S. 'Albatross' off the coast of Southern California in 1904. III. Euphrosynidae to Goniadidae. Proceedings of the academy of Natural History of Philadelphia 63: 234-318.

Müller, F. 1806. Zoologica Danica seu Animalium Daniae et Norvegiae ruriorum ac minus notorum, Descriptions et Historia. Havniae $160 \mathrm{p}$.

Müller, F. 1858. Einiges uber die Annelidenfauna der Insel Santa Catharina an der Brasilianischen Kuste. Archiv für Naturgeschichte 24: 211-220. 


\section{LISTS OF SPECIES}

Nonato, E. 1963. Poecilochaetus australis sp. nov. (Annelida, Polychaeta). Neotropica 9: 17-26.

Nonato, E. 1966. Sternaspis capillata sp. n. (Annelida, Polychaeta). Boletim do Instituto Oceanográfico de São Paulo 15: 79-83.

Nonato, E. F. 1981. Contribuição ao conhecimento dos anelídeos poliquetos bentônicos da plataforma continental brasileira, entre Arroio Chui e Cabo Frio. Unpublished thesis, Instituto Oceanográfico, Universidade de São Paulo, São Paulo, Brazil.

Nonato, E. F., G. A. Bolívar and P. C. Lana. 1986. Laonice branchiata a new species of Spionidae (Annelida: Polychaeta) from Southeastern Brazilian coast. Nerítica 1(3): 21-26.

Oersted, A. S. 1843. Annulatorum danicorum conspectus fasc. 1 Maricolae. Københaven.

Orensanz, J. M. and N. M. Gianuca. 1974. Contribuição ao conhecimento dos anelídos poliquetas do Rio Grande do sul, Brasil. I. lista sistemática preliminar e descrição de três novas espécies. Comunicações do Museu de Ciências da PUCRGS 4: 1-37.

Orensanz, J. M. 1973. Los anelidos poliquetos de la província biogeografica Argentina. III. Dorvilleidae. Physis 32: 325-342.

Orensanz, J. M. 1974. Los anelidos poliquetos de la província biogeografica Argentina. V. Onuphidae. Physis 33: 75-122.

Pallas, P. S. 1776. Miscellanea zoologica quibus novae imprimis atque obscurae Animalium species describuntur et observationibus iconibusque illustrantur. Den Haag, Hagi Comitum.

Paxton, H. 1974. Contribution to the study of the Australian Nephtyidae (Polychaeta). Record of Australian Museum 29: 197-208.

Paxton, H. and J. H. Bailey-Brock. 1986. Diopatra dexiognatha, a new species of Onuphidae (Polychaeta) from Oahu, Hawaian Islands. Pacific Science 40: 1-6.

Perkins, T. H. 1980. Review of the species previously referred to Ceratonereis mirabilis, and descriptions of new species of Ceratonereis, Nephtys, and Goniada (Polychaeta). Proceedings of the biological Society of Washington 93: 1-49.

Petersen, J. A. and E. S. Fanta. 1969. On two new species of Mesochaetopterus (Polychaeta) from the Brazilian coast. Beitrage zur Neotropical Fauna 6: 120-136.

Pettibone, M. H. 1957. A new polychaetous annelid of the family Paraonidae from the North Atlantic. Journal of Washington Academy of Science 47: 354-356.

Pettibone, M. H. 1962. New species of polychaete worms (Spionidae: Spiophanes) from the east and west coast of North America. Proceedings of the biological Society of Washington 75: 77-88.

Pettibone, M. H. 1965. Two new species of Aricidea (Polychaeta, Paraonidae) from Virginia and Florida, and redescription of Aricidea fragilis Webster. Proceedings of the biological Society of Washington 78 : 127-140.

Pettibone, M. H. 1966. Revision of the Pilargidae (Annelida:Polychaeta), including descriptions of new species, and redescriptions of the pelagic Podarmus ploa Chamberlain (Polynoidae). Proceedings of United Sates Natural Museum 118: 155-207.

Pflugfelder, O. 1933. Landpolychaten aus Niederlandisch-Indien. Zoologischer Anzeiger 105: 65-76.

Quatrefages, A. 1895. Histoire naturelle des anneles marines et d’eau douce. Annélides et gephiriens. Libraire Encyclopédique de Roret 2: 1-58.

Radashevsky, V. I. 2005. On adult and larval morphology of Polydora cornuta Bosc, 1802 (Annelida: Spionidae). Zootaxa 1064: $1-24$.

Radashevsky, V. I., and H. L. Hsieh. 2000. Pseudopolydora (Polychaeta: Spionidae) species from Taiwan. Zoological Studies 39: 218-235.

Rathke, H. 1843. Beitrage zur Fauna Norwegens. Nova Acta der Kaiserlichen LeopoldCarolin Deutschen Akademie der Naturforscher 20: 1-264.

Renier, S. A. 1804. Prospetto della Classe dei Vermi, nominati e ordinati secondo il Sistema di Bosc. Padua.

Reish, D. J. 1959. New species of Spionidae (Annelida, Polychaeta) from Southern California. Bulletin of South Califórnia Academy of Science 58: 11-16.

Ribeiro, Z. 2004. Análise cladística de Poecilochaetidae Hannerz, 1956 (Annelida, 


\section{LISTS OF SPECIES}

Polychaeta). Unpublished Thesis. Departamento de Zoologia, Universidade Federal do Paraná, Curitiba, Brazil.

Rioja, E. 1946. Estudios anelidologicos. XV. Nereidos de agua salobre de los esteros del litoral del Golfo de Mexico. Anales del Instituto de Biologia, Mexico 17: 205-214.

Risso, A. 1826. Histoire naturelle des principales productions de l'Europe meridionale et particulierement de celles des environs de Nice et des Alpes Maritimes. Paris.

Rizzo, A. 2002. Anelídeos Poliquetas da Plataforma Externa e Talude Continental ao Largo da Costa do Estado de São Paulo. Unpublished Thesis, Universidade do Estado de São Paulo, São Paulo, Brazil.

Rizzo, A. E. and A. C. Z Amaral. 2004a. Bathyglycinde profunda (Hartman and Fauchald, 1971) (Polychaeta, Goniadidae): new combination. Revista Brasileira de Zoologia 21(4): 937-942.

Rizzo, A. E. and A. C. Z. Amaral. 2004b. Progoniada and Goniadella (Annelida: Polychaeta: Goniadidae) of the outer continental shelf and slope off SouthEastern Brazil. Journal of the marine Biological Association of the United Kingdom 84: 47-58.

Rullier, F. and L. Amoureux. 1979. Campagne de la Calypso au large des côtes Atlantiques de l'Amerique du Sud (1961-1962). I. 33. Annelides Polychètes. Annales de l'Institute Océanographique 55: 145-205.

Salazar-Vallejo, S. I. 1990. Redescriptions of Sigambra grubii Müller, 1858 and Hermundura tricuspis Müller, 1858 from Brazil and designation of neotypes (Polychaeta, Pilargidae). Journal of Natural History 24: 507-517.

Santos, C. S. G. 2001. Revisão e análise cladística da subfamília Gymnonereidinae (Nereididae, Polychaeta). Unpublished Thesis. Departamento de Zoologia, Universidade Federal do Paraná, Curitiba, Brazil.

Santos, C. S. G. and A. S. Y. Mackie (in preparation). Two new species of Poecilochaetus from Paranaguá Bay (Southeast, Brazil).

Sars, M. 1851. Beretning om en i Sommeren 1849 foretagen zoologisk Reise i Lofoten og
Finmarken. Nyt Magazin for Naturvidenskaberne 6: 121-211.

Savigny, J. C. in J. P. de Lamarck. 1818. Histoire Naturelle des animaux sans vertèbres, presentant les caractères généraux et particuliers de ces animaux, leur distribution, leurs classes, leurs familles, leurs genres, et la citation des principales espèces qui s'y rapportent; précédes d'une. Paris.

Schmarda, L. K. 1861. Neue wirbellose thiere beobachtet und gesammelt auf einer Reise um die Erde 1853 bis 1857. Vol. 1. Turbellarian, Rotatorien und Anneliden. Leipzig, Wilhelmann.

Sene-Silva, G. 2003. Revisão taxonômica e análise cladística de Owenia (Polychaeta, Annelida). Unpublished Thesis. Departamento de Zoologia, Universidade Federal do Paraná, Curitiba, Brazil.

Solis-Weiss, V., K. Fauchald and A. Blankensteyn. 1991. Trichobranchidae (Polychaeta) from shallow warm water areas in the western Atlantic Ocean. Proceedings of Biological Society of Washington 104: 147-158.

Southern, R. 1914. Clare Island Survey. Archiannelida and Polychaeta. Proceedings of the Royal Irish Academy 31: 1-160.

Southern, R. 1921. Polychaeta of the Chilka Lake and also of fresh and brackish waters in other parts of India. Memoirs of the Indian Museum 5: 563-659.

Steiner, T. M. and C. S. G. Santos. 2004. A new species of Neanthes (Annelida, Polychaeta, Nereididae) from Brazil an some remarks on Neanthes bruaca Lana and Sovierzoski, 1987. Beaufortia 54: 39-57.

Steiner, T. M. 2006. Estudo taxonômico da família Onuphidae (Annelida, Polychaeta) das regiões Sudeste e Sul do Brasil. Unpublished thesis. Universidade de São Paulo, São Paulo, Brazil.

Temperini, M. T. 1981. Sistemática e distribuição dos poliquetas errantes da plataforma continental brasileira entre as latitudes $23^{\circ} 05^{\prime} \mathrm{S}$ e $30^{\circ} 00^{\prime} \mathrm{S}$. Unpublished thesis, Universidade de São Paulo, São Paulo, Brazil.

Treadwell, A. L. 1906. Polychaetous annelids from the Hawaian Islands, collected by Stremaer Albatross in 1902. Bulletin of the 


\section{LISTS OF SPECIES}

United States Fishires Communication 23: 1145-1181.

Treadwell, A. L. 1923. Duas novas espécies de anelídeos poliquetos do gênero Nereis. Revista do Museu Paulista 13: 1237-1243.

Verrill, A. E. and R. Rathbun. 1879. List of marine invertebrata from the New England coast, distributed by the U. S. Commissiion of Fish and Fisheries. Proceedings of the United States of Natural Museum 2: 227232.

Verrill, A. E. 1873. Results of recent dredging expeditions on the coast of New England. American Journal of Science and Arts 5: 181.

Verrill, A. E. 1900. Additions to the Turbellaria, Nemertina, and Annelida of the Bermudas, with revisions of some New England genera and species. Transactions of the Connecticut Academy of Arts and Sciences 10: 595-671.

Webster, H. E. 1879. The Annelida Chaetopoda of New Jersey. Annual Reports of the New York State Museum of Natural History 32: 101-128.

Webster, H. E. 1879. The Annelida Chaetopoda of the Virginian coast. Transactions Albany Institute New York 9: 202-272.

Webster, H. E. 1884. Annelida from Bermuda collected by G. Brown Goode. Bulletins of the United States of Natural Museum 25: 307-327.

Woodwick, K. H. 1953. Polydora nuchalis, a new species of Polychaetous Annelid from California. Journal of Washington Academy of Sciencia 43: 381-383.

\section{Additional references}

Alves, P. R. P. 1997. Efeitos da vegetação e da predação sobre as associações macrobênticas de uma planície de maré da Baía de Paranaguá (Paranaguá, Brasil). Unpublished thesis, Departamento de Zoologia, Universidade Federal do Paraná, Curitiba, Brazil.

Barros, F., C. A. Borzone and S. Rosso. 2001. Macroinfauna of six beaches near Guaratuba Bay, Southern Brazil. Brazilian Archives of Biology and Technology 44: 351-364.
Blankensteyn, A. 2003. Estrutura e análise experimental do funcionamento das associações da macrofauna bêntica do manguezal e marisma da Gamboa Perequê, Pontal do Sul, PR. Unpublished thesis, Departamento de Zoologia, Universidade Federal do Paraná, Curitiba, Brazil.

Borzone, C. A., and J. R. B. Souza. 1997. Estrutura da macrofauna bentônica no supra, meso e infralitoral de uma praia arenosa do sul do Brasil. Oecologia brasiliensis 3: 197-212.

Borzone, C. A., K. C. Gutseit and C. Soares. 1999. Sedimentos e macrofauna bentônica da zona de águas rasas da praia de Matinhos, Paraná, Brasil. Atlântica 21: 4358.

Borzone, C. A., J. R. B. Souza and C. Soares. 1996. Morphodynamic influence on the structure of inter and subtidal macrofaunal communities of subtropical sandy beaches. Revista Chilena de Historia Natural 69: 565-577.

Brogim, R. A. 2001. Variabilidade de espaçotemporal da macroinfauna bêntica de manguezais das Baías de Paranaguá e Antonina (Paraná - Brasil). Unpublished thesis. Departamento de Zoologia, Universidade Federal do Paraná, Curitiba, Brazil.

Camargo, M. G. 2001. Análise experimental das interações biológicas da macrofauna e meiofauna bêntica de manguezais das Baías de Paranaguá e Antonina - PR. Unpublished thesis. Departamento de Zoologia. Universidade Federal do Paraná, Curitiba, Brazil.

Couto, E. C. G. 1996. Estrutura espaço-temporal da comunidade macrobêntica da planície intertidal do Saco do Limoeiro, Ilha do Mel (Paraná, Brasil). Unpublished thesis. Departamento de Zoologia. Universidade Federal do Paraná, Curitiba, Brazil.

Faraco, L. F. D. 2002. Análise experimental dos processos de recolonização macrobêntica em um manguezal subtropical. Unpublished thesis. Departamento de Zoologia. Univrsidade Federal do Paraná, Curitiba, Brazil. 


\section{LISTS OF SPECIES}

Faraco, L. F. D. and P. C. Lana. 2003. Response of polychaetes to oil spills in natural and defaunated subtropical mangrove sediments from Paranaguá Bay (SE Brazil). Hydrobiologia 496: 321-328.

Lana, P. C. and C. Guiss. 1991. Influence of Spartina alterniflora on structure and temporal variability of macrobenthic associations in a tidal flat of Paranaguá Bay (Southeastern Brazil). Marine Ecology Progress Series 73: 231-244.

Lana, P. C. 1986. Macrofauna bêntica de fundos sublitorais não consolidados da Baía de Paranaguá (Paraná). Nerítica 1: 79-89.

Lana, P. C., M. V. O. Almeida, M. Freitas, C. Couto, E. Conti, L. Gonzales-Peronti, A. Giles, A. Lopes, M. Silva and L. Pedroso. 1989. Estrutura espacial de associações macrobênticas sublitorais da Gamboa Perequê (Pontal do Sul, Parana). Neritica 4: 119-136.

Lana, P. C., E. C. G. Couto and M. V. O. Almeida. 1997. Polychaete distribution and abundance in intertidal flats of Paranaguá Bay (SE Brazil). Bulletin of Marine Science 60: 433-442.

Lana, P. C., C. Guiss and S. T. Disaró. 1991. Seasonal variation of biomass and production dynamics for above and below ground components of a Spartina alterniflora marsh in the euhaline sector of Paranaguá Bay (SE Brazil). Estuarine and Coastal Shelf Science 32: 231-241.

Netto, S. A. 1993. Composição, distribuição e variabilidade sazonal da macrofauna bêntica de marismas e bancos não-vegetados da Baía de Paranaguá (Paraná, Brasil). Unpublished thesis. Departamento de Zoologia. Universidade Federal do Paraná, Curitiba, Brazil.
Netto, S. A. and P. C. Lana. 1995. Zonação e estratifição da macrofauna bêntica em um banco areno-lodoso so setor euhalino de alta energia da Baía de Paranaguá (Paraná, Brasil). Iheringia 79: 37-27.

Netto, S. A. and P. C. Lana. 1997. Influence of Spartina alterniflora on superficial sediment characteristics of tidal flats in Paranaguá Bay (South-Eastern Brazil). Estuarine Coastal and Shelf Science 44: 1-8.

Netto, S. A. and P. C. Lana. 1994. Effects of sediment disturbance on the structure of benthic fauna in a subtropical tidal creek of Southeastern Brazil. Marine Ecology Progress and Series 106: 239-247.

Pagliosa, P. R. and P. C. Lana. 2000. Population dynamics and secondary production of Nereis oligohalina (Nereididae: Polychaeta) from a subtropical marsh in Southeast Brazil. Bulletin of Marine Scince 67: 268259.

Sovierzoski, H. H. 1991. Estrutura temporal da comunidade macrobentônica da Foz do Rio Maciel, Baía de Paranaguá, Paraná. Unpublished thesis. Departamento de Zoologia. Universidade Federal do Paraná, Curitiba, Brazil.

Souza, J. R. B. and C. A. Borzone. 2000. Population dynamics and secondary production of Scolelepis squamata (Polychaeta: Spionidae) in an exposed sandy beach, southern Brazil. Bulletin of Marine Science 67: 221-233.

Souza, J. R. B. 1998. Produção secundária da macrofauna bentênica da praia de Atami Paraná - Brasil. Unpublished thesis. Departamento de Zoologia. Universidade Federal do Paraná, Curitiba, Brazil.

Received May 2006

Accepted October 2006

Published online October 2006 